\title{
Mortality and Disease Severity Among COVID-19 Patients Receiving Renin-Angiotensin System Inhibitors: A Systematic Review and Meta-analysis
}

\author{
Syed Shahzad Hasan ${ }^{1}$ [D Chia Siang Kow ${ }^{2} \cdot$ Muhammad Abdul Hadi $^{3} \cdot$ Syed Tabish Razi Zaidi ${ }^{4,5}$. \\ Hamid A. Merchant ${ }^{1}$
}

Accepted: 29 August 2020 / Published online: 12 September 2020

(c) Springer Nature Switzerland AG 2020

\begin{abstract}
Introduction The use of renin-angiotensin system (RAS) inhibitors, including angiotensin-converting enzyme inhibitors (ACEIs) and angiotensin receptor blockers (ARBs), was alleged to cause a more severe course of novel coronavirus disease 2019 (COVID-19).

Methods We systematically reviewed the published studies to assess the association of RAS inhibitors with mortality as well as disease severity in COVID-19 patients. A systematic literature search was performed to retrieve relevant original studies investigating mortality and severity (severe/critical disease) in COVID-19 patients with and without exposure to RAS inhibitors.

Results A total of 59 original studies were included for qualitative synthesis. Twenty-four studies that reported adjusted effect sizes (24 studies reported mortality outcomes and 16 studies reported disease severity outcomes), conducted in RAS inhibitor-exposed and unexposed groups, were pooled in random-effects models to estimate overall risk. Quality assessment of studies revealed that most of the studies included were of fair quality. The use of an ACEI/ARB in COVID-19 patients was significantly associated with lower odds (odds ratio $[\mathrm{OR}]=0.73,95 \%$ confidence interval $[\mathrm{CI}] 0.56-0.95 ; n=18,749$ ) or hazard (hazard ratio $[\mathrm{HR}]=0.75,95 \% \mathrm{CI} 0.60-0.95 ; n=26,598)$ of mortality compared with non-use of ACEI/ARB. However, the use of an ACEI/ARB was non-significantly associated with lower odds ( $\mathrm{OR}=0.91,95 \% \mathrm{CI} 0.75-1.10 ; n=7446)$ or hazard $(\mathrm{HR}=0.73,95 \% \mathrm{CI} 0.33-1.66 ; n=6325)$ of developing severe/critical disease compared with non-use of an ACEI/ARB.

Discussion Since there was no increased risk of harm, the use of RAS inhibitors for hypertension and other established clinical indications can be maintained in COVID-19 patients.
\end{abstract}

Electronic supplementary material The online version of this article (https://doi.org/10.1007/s40256-020-00439-5) contains supplementary material, which is available to authorized users.

Syed Shahzad Hasan

s.hasan@hud.ac.uk

1 Department of Pharmacy, University of Huddersfield, Huddersfield HD1 3DH, UK

2 School of Postgraduate Studies, International Medical University, Kuala Lumpur 57000, Malaysia

3 Institute of Clinical Sciences, University of Birmingham, Edgbaston, Birmingham B15 2TT, UK

4 School of Healthcare, University of Leeds, Leeds LS2 9JT, UK

5 Leeds Teaching Hospitals NHS Trust, Leeds LS2 9JT, UK

\section{Key Points}

The use of renin-angiotensin system (RAS) inhibitors is alleged to cause a more severe course of coronavirus disease 2019 (COVID-19).

We systematically reviewed the published studies to assess the association of RAS inhibitors with mortality as well as disease severity in COVID-19 patients.

The meta-analyses favoured the continued use of RAS inhibitors in terms of risk of mortality and severe/critical COVID-19 disease. 


\section{Introduction}

Cardiovascular diseases including hypertension remain one of the most significant comorbidities identified in patients with novel coronavirus disease 2019 (COVID-19) [1-6]. The presence of cardiovascular diseases including hypertension tends to portend a more severe course of illness in patients hospitalized with COVID-19 [1-6]. Some researchers were quick to link such an association with the use of renin-angiotensin system (RAS) inhibitors, including angiotensinconverting enzyme inhibitors (ACEIs) and angiotensin type I receptor blockers (ARBs), among patients with cardiovascular disease $[7,8]$.

The causative pathogen for COVID-19, namely severe acute respiratory syndrome coronavirus 2 (SARS-CoV-2), binds to the ACE2 receptor on host cells to gain entry [9]. In several animal models and human studies, the expression of ACE2 is increased with the chronic administration of RAS inhibitors, which suggests a possibility for the increased susceptibility to SARS-CoV-2 infection and a more severe course of illness with the use of RAS inhibitors [10-17]. Conversely, there are studies [18-23] that reported no association between increased expression of ACE2 receptor and the use of RAS inhibitors. Interestingly, a protective role of ACE2 has been suggested in the mitigation of coronavirus-induced lung injury [24, 25]. In addition, it has been theoreticized that RAS inhibitors can be used as a potential therapy for COVID-19 as both ACE and its product angiotensin II, for which their downstream effects are inhibited by RAS inhibitors, seem to promote lung injury [25]. It has been demonstrated that the coronavirus spike protein binds to ACE2, leading to ACE2 downregulation, which in turn results in the retention of angiotensin II due to a lack of its clearance/conversion to angiotensin (1-7) by ACE2 and leads to vasoconstriction of pulmonary vessels, increased pulmonary vascular permeability, and increased inflammation due to overactivity of the vasoconstrictive ACE/angiotensin II pathway $[25,26]$. Therefore, users of RAS inhibitors should, in theory, be protected from acute respiratory distress syndrome (ARDS), the primary cause of mortality in COVID-19 [3, 4, 28-34]. Nonetheless, such a lung-protective mechanism is not firmly established in human trials.

Several learned cardiovascular societies have discredited the association between RAS inhibitors and the increased susceptibility to COVID-19 or associated poor outcomes and encouraged clinicians and patients to continue the use of prescribed RAS inhibitors in hypertension or other established indications [27]. In addition, several observational studies have since reported clinical outcomes among patients with COVID-19 receiving RAS inhibitors [35-93]. As this issue remains one of the most debated subjects among clinicians and researchers amid the COVID-19 pandemic, we systematically reviewed the data from available studies to date to critically examine the association of RAS inhibitors use with mortality and disease severity in COVID-19.

\section{Methods}

\subsection{Scope of Review: Eligibility Criteria}

This systematic review was performed with a protocol in accordance with the Preferred Reporting Items for Systematic Review and Meta-Analyses (PRISMA) statements [94]. To systematically review the mortality and disease severity in COVID-19 patients receiving RAS inhibitors, a literature search was performed using scientific databases to identify original studies (prospective or retrospective in design) published in full between 1 January 2020 and 31 August 2020. The eligibility criteria, which were defined prospectively, are provided in Box 1.

\subsection{Box 1: Eligibility criteria}

Original, observational (prospective or retrospective) studies Included patients with coronavirus disease 2019 (COVID-19)

Documented use of either angiotensin-converting enzyme (ACE) inhibitors or angiotensin receptor blockers (ARBs)

Reported frequency, percentage, and/or adjusted estimate of mortality or disease severity and/or adverse clinical outcomes (septic shock, admission to intensive care units) associated with COVID-19

From any region or language

\subsection{Information Sources and Search Strategy}

Two authors (SSH and CSK) independently performed a systematic literature search in PubMed, Google Scholar, and two preprint repositories (medRxiv and SSRN) without language restriction up to 19th August 2020. The medical literature was searched using the following search terms: angiotensin-converting enzyme or ACE or ACE inhibitor or angiotensin receptor blocker or ARB or renin-angiotensin-system or renin-angiotensin-system or RAS inhibitor or renin-angiotensin-aldosterone or RAA inhibitor or RAAS inhibitor) AND COVID-19 OR novel coronavirus OR severe acute respiratory syndrome OR SARS-CoV-2. The search was limited to original observational studies (prospective or retrospective), involving human subjects, and published in any language. However, studies in the Chinese language were only assessed by CSK (native Chinese speaker). The titles and abstracts of the resulting articles were first examined to exclude irrelevant studies. Subsequently, the full 
texts of the remaining articles were read to determine if studies met the eligibility criteria in full. Bibliographies of retrieved articles were also reviewed to search for additional studies. Differing decisions were resolved by mutual consensus. Articles were excluded if they contained no original data (narrative reviews, letters, opinions, and comments) or reported a combined severity and mortality endpoint without individual presentation of severity and mortality data.

\subsection{Data Extraction}

One of the authors (CSK) extracted data independently on a Microsoft Excel spreadsheet (XP Professional edition; Microsoft, Redmond, Washington, USA) that was verified by the second reviewer (SSH). In the case of disagreement, a third author was involved to resolve, by consensus, any discrepancies with respect to the relevance of the sources. The following data were collected for each study: the name of the first author; country; publication year; study design; the number of subjects; the age of the subjects; the presence of hypertension; the frequency of deaths; the frequency of severe/critical disease; adjusted estimates; and confounders.

\subsection{Assessment of Quality of Included Studies}

The methodological quality of the eligible studies was examined using the Newcastle-Ottawa Scale for cohort studies [95]. The Newcastle-Ottawa Scale is easy to use with its star rating system and is considered reliable to measure biases in cohort studies. Each of the selected cohort studies was evaluated for selection of study group ( $0-4$ stars), comparability or quality of adjustment for confounding factors (0-2 stars), and ascertainment of the outcome of interest (0-3 stars), with a maximum of nine stars representing the highest methodological quality. Studies with a Newcastle-Ottawa Scale score of $>7$ were regarded as high quality.

\subsection{Data Synthesis and Analysis}

The reported odds ratios (ORs) and hazard ratios (HRs) that had been adjusted for potential covariates in the respective original studies and the corresponding $95 \%$ confidence intervals (CIs) were extracted and pooled in a random-effects model to estimate the association between the use of ACEIs/ ARBs and the risk of mortality and severe/critical illness in COVID-19 patients. If a study reported the estimates from different multivariable models, the most extensively adjusted estimate in terms of the number of covariates was extracted. However, in the presence of different multivariable models adjusted for the same number of covariates, the model containing the most clinically meaningful covariates was extracted for the pooled analysis. A random-effects model was employed since we assumed that the treatment effect was not the same across all the studies included in the analysis. Cochran's $Q$ heterogeneity test ( $Q$ test) and a related metric, the $I^{2}$, were used to evaluate heterogeneity. We used a $p$ value of 0.10 and an $I^{2}$ value of $50 \%$ as the cut-off points for statistically significant heterogeneity. Publication bias was examined through visual inspection of the funnel plot for symmetry. Four different subgroup analyses were conducted: (1) the first subgroup analysis considered only studies that included, exclusively, hypertensive COVID-19 patients in their analysis, to determine the effect of ACEIs/ ARBs in this patient population; (2) the second subgroup analysis was based on the region where the studies were performed to determine the presence of heterogeneity in the effect of ACEIs/ARBs across different regions globally; (3) the third subgroup analysis considered only studies that reported separate estimates for the use of ACEIs and ARBs, to determine the presence of differential effects between the two classes of RAS inhibitors; and (4) the fourth subgroup analysis was based on the different definitions of severe/ critical outcome in COVID-19, to determine the presence of heterogeneity in the effect of ACEIs/ARBs according to the definitions of severe/critical outcome. All meta-analytical calculations were performed using Meta XL, version 5.3 (EpiGear International, Queensland, Australia).

\section{Results}

\subsection{Characteristics of the Included Studies}

Our literature search yielded 10,481 unique titles. A PRISMA flowchart [89] of the literature search and study selection with the number of studies at each stage is presented in Fig. 1. After deduplication and application of the eligibility criteria, 523 relevant full-text articles were examined for inclusion. Of these, 464 studies were excluded for three reasons: no original data, combined mortality and severity endpoint, or retraction. A total of 59 original studies [35-93] that compared the mortality and/or severity outcomes between COVID-19 patients receiving an ACEI/ $\mathrm{ARB}$ and their counterparts not receiving an ACEI/ARB were finally shortlisted for the qualitative synthesis.

The characteristics of the shortlisted studies are summarized in Table 1. Among shortlisted studies, 23 studies were from China [36-58], nine studies were from Italy [64-72], ten studies were from the United States [83-92], four studies were from South Korea [75-78], three studies were from France [60-62], two studies were from Spain [79, 80], one study each was from Belgium [35], Denmark [59], Hong Kong [63], Kuwait [73], Singapore [74], Turkey [81], and the United Kingdom [82]; and one study included data from 38 countries [93]. There were 24 studies that included 


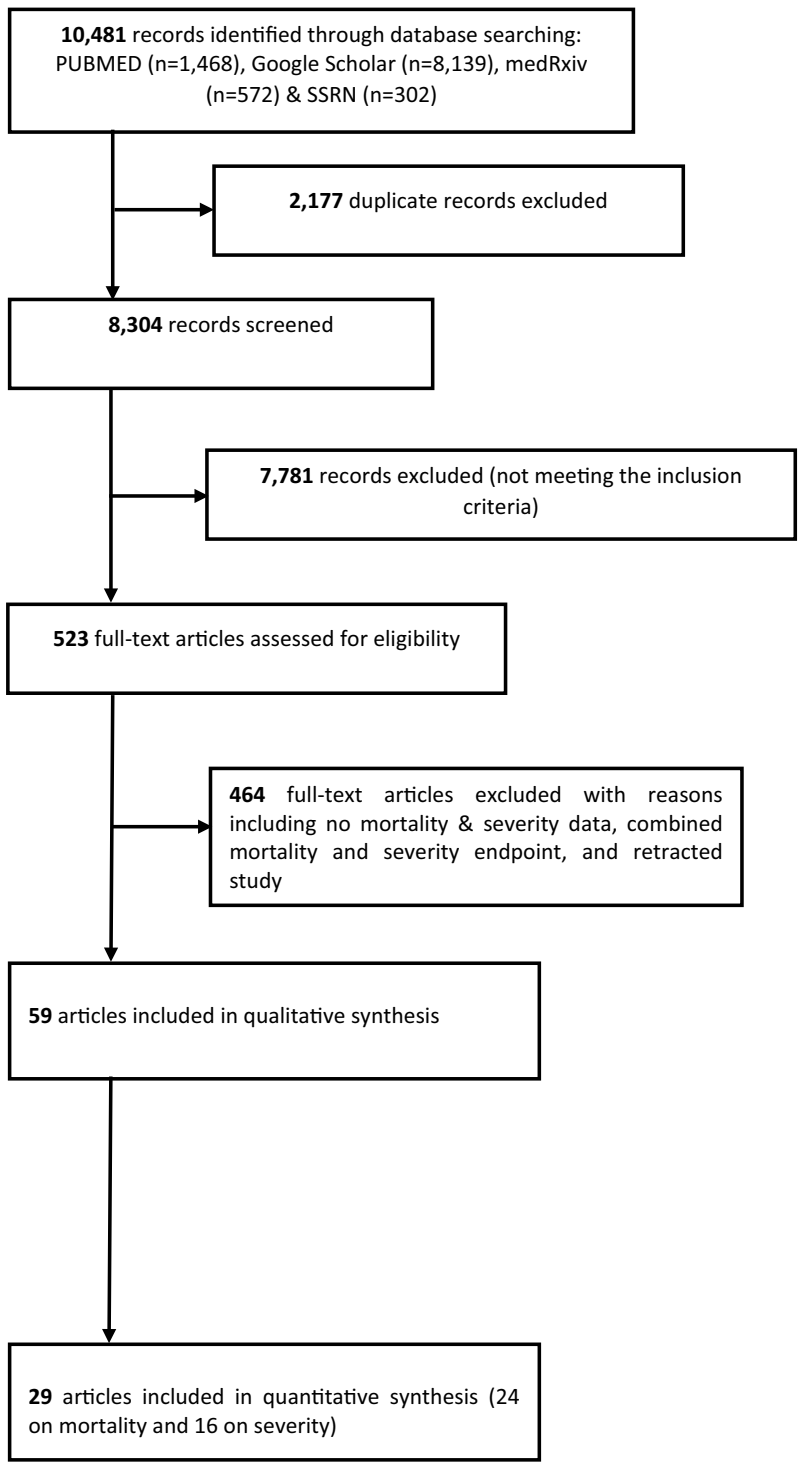

Fig. 1 Study selection process (Preferred Reporting Items for Systematic Review and Meta-Analyses [PRISMA])

hypertensive patients exclusively (100\%) [36, 38, 39, 43-45, $47,48,50-52,54,56,58,66,71,72,75,78,81,87-89,91]$.

The comparison of mortality and clinical severity outcomes between ACEI/ARB users and non-ACEI/ARB users with COVID-19 is summarized in Table 2. There were 50 studies [35-38, 41-45, 48-51, 53-59, 61, 62, 64-82, 85-93] and 36 studies [35, 36, 39-41, 43-53, 55, 56, 59-63, 66, 71, $74,75,78,80,83-85,89-92]$, respectively, that reported mortality outcomes and clinical severity outcomes among COVID-19 patients with and without the use of ACEIs/ ARBs. Among 50 studies that reported mortality outcomes, 24 studies [35, 36, 54, 56, 57, 59, 64, 66, 67, 69, 70, 72, $74-78,80,81,86,88,89,92,93]$ provided adjusted mortality estimates with the use of an ACEI/ARB relative to the non-use of an ACEI/ARB. Out of 36 studies that reported outcomes on clinical severity, 16 studies [35, 40, 52, 56, 59, $61,63,66,74,75,78,80,84,89,90,92]$ provided adjusted estimates for severe/critical disease with the use of an ACEI/ $\mathrm{ARB}$ relative to the non-use of an ACEI/ARB.

\subsection{Mortality Associated with the Use of RAS Inhibitors in COVID-19 Patients}

There were 24 studies $[35,36,54,56,57,59,64,66,67$, $69,70,72,74-78,80,81,86,88,89,92,93]$ that provided adjusted estimates on the risk of mortality among ACEI/ ARB and non-ACEI/ARB users. The Newcastle-Ottawa Scale [95] was used for the quality assessment of all studies that provided adjusted estimates and were included in the meta-analysis of the risk of mortality associated with the use of ACEIs/ARBs (Supplementary Table S1, see the Electronic Supplementary Material). Of the 24 original studies included, only two studies, by Zhang et al. [56] and Zhou et al. [57], were deemed 'good', with a score of 7 points. The remaining 22 studies $[35,36,54,59,64,66,67,69$, $70,72,74-78,80,81,86,88,89,92,93]$ were regarded as 'fair', with scores of 4-6 points. No studies were considered 'poor', i.e. scored less than 4 points in the quality assessment. The main quality issue often noticed was the comparison of cohorts, with inadequate adjustment for the confounders that may influence the estimated risk of mortality associated with the use of ACEIs/ARBs in 21 studies [35, $36,54,59,64,66,67,69,70,72,74,76-78,80,81,86,88$, $89,92,93]$. Another major issue detected during the quality assessment was the inability to ascertain exposure to ACEIs/ ARBs during the course of illness in 19 studies, where a possibility of ACEIs/ARBs discontinuation upon COVID-19 diagnosis could not be ruled out based on the study design $[35,36,54,59,66,67,69,70,72,74-78,80,86,88,92$, 93]. Representativeness of the exposed cohort could not be established in 16 studies [36, 54, 56, 57, 59, 64, 67, 69, 72, $74,81,86,88,89,92,93]$ that included hospitalized patients only. In 13 studies [35, 59, 67, 72, 74-76, 78, 80, 81, 86, $88,93]$, it was unclear whether the entire included cohort of patients was followed until discharge/death.

Since Selçuk et al. [81] provided mortality estimates with a very wide $\mathrm{CI}(\mathrm{OR}=3.66$; 95\% CI $1.11-18.18)$, we excluded this study from our meta-analysis. In a pooled analysis of the 12 remaining original studies that provided adjusted ORs, with 18,749 COVID-19 patients being analyzed [35, 64, 66, 75, 76, 78, 80, 86, 88, 89, 92, 93], the use of an ACEI/ARB was significantly associated with lower odds of mortality compared to non-use of an ACEI/ARB (Fig. 2; pooled OR $=0.73,95 \%$ CI $0.56-0.95$ ). In a separate pooled analysis of 11 studies that provided adjusted HRs, with 26,598 COVID-19 patients being analyzed [36, 54, 56, $57,59,67,69,70,72,74,77]$, the use of an ACEI/ARB was significantly associated with lower risk of mortality 
Table 1 Characteristics of the included studies

\begin{tabular}{|c|c|c|c|c|c|}
\hline Study & Study design & Origin & $\begin{array}{l}\text { Total } \\
\text { number of } \\
\text { patients }^{\mathrm{a}}\end{array}$ & $\mathrm{Age}^{\mathrm{b}}$ & $\begin{array}{l}\text { Proportion of patients } \\
\text { with hypertension }(\%)\end{array}$ \\
\hline Amaouche et al. ${ }^{\mathrm{c}}$ [35] & $\begin{array}{l}\text { Retrospective, single } \\
\text { centre }\end{array}$ & Brussels, Belgium & 299 & $\begin{array}{l}\text { Non-ACEI/ARB } \\
\text { users }=63 \text { (Mean) }\end{array}$ & 42.1 \\
\hline Chen $\mathrm{C}$ et al. [36] & $\begin{array}{l}\text { Retrospective, single } \\
\text { centre }\end{array}$ & Wuhan, China & 1182 & Non-hypertensive $=54$ & 100 \\
\hline Chen $\mathrm{M}$ et al. ${ }^{\mathrm{c}}$ [37] & $\begin{array}{l}\text { Retrospective, single } \\
\text { centre }\end{array}$ & Wuhan, China & 123 & $\begin{array}{l}\text { Discharged patients }=53 \\
\text { Death patients }=72\end{array}$ & 33.3 \\
\hline Chen Y et al. [38] & $\begin{array}{l}\text { Retrospective, single } \\
\text { centre }\end{array}$ & Wuhan, China & 71 & $\begin{array}{l}\text { Non-ACEI/ARB } \\
\text { users }=67\end{array}$ & 100 \\
\hline Feng Y et al. [39] & Retrospective, multicentre & China & 113 & All patients $=53$ & 100 \\
\hline Feng $\mathrm{Z}$ et al. ${ }^{\mathrm{c}}[40]$ & Retrospective, multicentre & China & 65 & $\begin{array}{l}\text { ACEI/ARB users }=57 \\
\text { Non-ACEI/ARB } \\
\text { users }=63\end{array}$ & 14.5 \\
\hline Gao et al. [41] & $\begin{array}{l}\text { Retrospective, single } \\
\text { centre }\end{array}$ & Wuhan, China & 2877 & $\begin{array}{l}\text { Hypertensive }=64 \\
\text { Non-hypertensive }=55 \\
\text { (Mean) }\end{array}$ & 29.5 \\
\hline Guo et al. [42] & $\begin{array}{l}\text { Retrospective, single } \\
\text { centre }\end{array}$ & Wuhan, China & 187 & All patients $=59$ (Mean) & 32.6 \\
\hline Hu et al. [43] & Retrospective, multicentre & Zhejiang, China & 149 & $\begin{array}{l}\text { ACEI/ARB users }=56 \\
\text { Non-ACEI/ARB } \\
\text { users }=58\end{array}$ & 100 \\
\hline Huang et al. [44] & $\begin{array}{l}\text { Retrospective, single } \\
\text { centre }\end{array}$ & Wuhan, China & 50 & $\begin{array}{l}\text { ACEI/ARB users }=53 \\
\text { Non-ACEI/ARB } \\
\text { users }=68(\text { Mean })\end{array}$ & 100 \\
\hline Li J et al. [45] & $\begin{array}{l}\text { Retrospective, single } \\
\text { centre }\end{array}$ & Wuhan, China & 362 & $\begin{array}{l}\text { ACEI/ARB users }=65 \\
\text { Non-ACEI/ARB } \\
\text { users }=67\end{array}$ & 100 \\
\hline Li X et al. [46] & $\begin{array}{l}\text { Ambispective; single } \\
\text { centre }\end{array}$ & Wuhan, China & 548 & All patients $=60$ & 30.3 \\
\hline Liu et al. ${ }^{\mathrm{c}}$ [47] & Retrospective, multicentre & China & 78 & All patients $=65$ (Mean) & 100 \\
\hline Meng et al. [48] & $\begin{array}{l}\text { Retrospective, single } \\
\text { centre }\end{array}$ & Shenzhen, China & 42 & All patients $=65$ & 100 \\
\hline Peng et al. [49] & $\begin{array}{l}\text { Retrospective, single } \\
\text { centre }\end{array}$ & Wuhan, China & 112 & All patients $=62$ & 82.1 \\
\hline Tan et al. [50] & $\begin{array}{l}\text { Retrospective, single } \\
\text { centre }\end{array}$ & Wuhan, China & 100 & $\begin{array}{l}\text { ACEI/ARB users }=67 \\
\text { Non-ACEI/ARB } \\
\text { users }=68\end{array}$ & 100 \\
\hline $\mathrm{Xu}$ et al. [51] & $\begin{array}{l}\text { Retrospective, single } \\
\text { centre }\end{array}$ & Wuhan, China & 101 & All patients $=65$ & 100 \\
\hline Yan et al. ${ }^{\mathrm{c}}[52]$ & Retrospective, multicentre & Zhejiang, China & 136 & All patients $=49$ & 100 \\
\hline Yang et al. [53] & $\begin{array}{l}\text { Retrospective, single } \\
\text { centre }\end{array}$ & Hubei, China & 251 & ACEI/ARB users $=65$ & 50.2 \\
\hline
\end{tabular}


Table 1 (continued)

\begin{tabular}{|c|c|c|c|c|c|}
\hline Study & Study design & Origin & $\begin{array}{l}\text { Total } \\
\text { number of } \\
\text { patients }^{\mathrm{a}}\end{array}$ & $\mathrm{Age}^{\mathrm{b}}$ & $\begin{array}{l}\text { Proportion of patients } \\
\text { with hypertension (\%) }\end{array}$ \\
\hline & & & & $\begin{array}{l}\text { Non-ACEI/ARB } \\
\text { users }=67\end{array}$ & \\
\hline Yuan et al. [54] & Retrospective, multicentre & Wuhan, China & 260 & $\begin{array}{l}\text { ACEI/ARB users }=67 \\
\text { Non-ACEI/ARB } \\
\text { users }=66\end{array}$ & 100 \\
\hline Zeng et al..$^{\mathrm{c}}[55]$ & $\begin{array}{l}\text { Retrospective, single } \\
\text { centre }\end{array}$ & Wuhan, China & 274 & $\begin{array}{l}\text { ACEI/ARB users }=64 \\
\text { Non-ACEI/ARB } \\
\text { users }=69\end{array}$ & 27.4 \\
\hline Zhang et al. [56] & Retrospective, multicentre & Hubei, China & 1128 & $\begin{array}{l}\text { ACEI/ARB users }=64 \\
\text { Non-ACEI/ARB } \\
\text { users }=64\end{array}$ & 100 \\
\hline Zhou F et al. [57] & Retrospective, multicentre & Hubei, China & 3572 & N/A & N/A \\
\hline Dauchet et al. ${ }^{\mathrm{c}}[60]$ & $\begin{array}{l}\text { Retrospective, single } \\
\text { centre }\end{array}$ & Lille, France & 187 & $\begin{array}{l}\text { Outpatient }=50 \\
\text { Inpatient }=58 \\
\text { ICU }=61 \text { (Mean) }\end{array}$ & N/A \\
\hline Liabeuf et al. [61] & Prospective, single centre & Amiens, France & 268 & All patients $=73$ & 56.7 \\
\hline Oussalah et al. [62] & $\begin{array}{l}\text { Retrospective, single } \\
\text { centre }\end{array}$ & Nancy, France & 147 & $\begin{array}{l}\text { ACEI/ARB users }=70 \\
\text { Non-ACEI/ARB } \\
\text { users }=63\end{array}$ & 49.6 \\
\hline Zhou J et al. ${ }^{\mathrm{c}}$ [63] & Retrospective, multicentre & Hong Kong & 976 & All patients $=34$ & 11.1 \\
\hline Cannata et al. [64] & $\begin{array}{l}\text { Retrospective, single } \\
\text { centre }\end{array}$ & Lombardy, Italy & 280 & - & - \\
\hline Conversano et al. [65] & $\begin{array}{l}\text { Retrospective, single } \\
\text { centre }\end{array}$ & Milan, Italy & 191 & $\begin{array}{l}\text { Discharged patients }=60 \\
\text { Death patients }=75 \\
\quad(\text { Mean })\end{array}$ & 50.2 \\
\hline \multirow[t]{2}{*}{ Felice et al. [66] } & $\begin{array}{l}\text { Retrospective, single } \\
\text { centre }\end{array}$ & Treviso, Italy & 133 & ACEI users $=73$ & 100 \\
\hline & & & & $\begin{array}{l}\text { ARB users }=69 \\
\text { Non-ACEI/ARB } \\
\text { users }=76(\text { Mean })\end{array}$ & \\
\hline Ferrante et al. [67] & $\begin{array}{l}\text { Retrospective, single } \\
\text { centre }\end{array}$ & Milan, Italy & 332 & All patients $=67$ & 54.1 \\
\hline Giacomelli et al. [68] & Prospective, single centre & Milan, Italy & 233 & All patients $=61$ & N/A \\
\hline Grasselli et al. [69] & Retrospective, multicentre & Milan, Italy & 3988 & All patients $=63$ & 41.2 \\
\hline Rossi et al. [70] & Prospective database & Reggio Emilia, Italy & 2362 & All patients $=63$ & 18.1 \\
\hline Sardu et al. [71] & Prospective, multicentre & Naples, Italy & 62 & All patients $=58$ (Mean) & 100 \\
\hline Tedeschi et al. [72] & Retrospective, multicentre & Italy & 311 & All patients $=76$ & 100 \\
\hline Ayed et al. [73] & $\begin{array}{l}\text { Retrospective, single } \\
\text { centre }\end{array}$ & Kuwait & 103 & All patients $=53$ & 35.0 \\
\hline Dalan et al. [74] & $\begin{array}{l}\text { Retrospective, single } \\
\text { centre }\end{array}$ & Singapore & 717 & - & 19.4 \\
\hline Choi et al. [75] & $\begin{array}{l}\text { Retrospective database } \\
\text { review }\end{array}$ & South Korea & 1250 & $\begin{array}{l}\text { ACEI/ARB users }=65 \\
\text { Non-ACEI/ARB } \\
\text { users }=68 \\
\text { (Mean) }\end{array}$ & 100 \\
\hline Jung $S$ et al. [76] & $\begin{array}{l}\text { Retrospective database } \\
\text { review }\end{array}$ & South Korea & 5179 & $\begin{array}{l}\text { ACEI/ARB users }=63 \\
\text { Non-ACEI/ARB } \\
\text { users }=42 \\
\text { (Mean) }\end{array}$ & 22.3 \\
\hline Lee et al. ${ }^{\mathrm{c}}[77]$ & $\begin{array}{l}\text { Retrospective database } \\
\text { review }\end{array}$ & South Korea & 8266 & $\begin{array}{l}\text { ACEI users }=69 \\
\text { ARB users }=64 \\
\text { Non-ACEI/ARB } \\
\text { users }=42 \\
\text { (Mean) }\end{array}$ & 19.0 \\
\hline
\end{tabular}


Table 1 (continued)

\begin{tabular}{|c|c|c|c|c|c|}
\hline Study & Study design & Origin & $\begin{array}{l}\text { Total } \\
\text { number of } \\
\text { patients }^{\mathrm{a}}\end{array}$ & $\mathrm{Age}^{\mathrm{b}}$ & $\begin{array}{l}\text { Proportion of patients } \\
\text { with hypertension (\%) }\end{array}$ \\
\hline Son et al. [78] & $\begin{array}{l}\text { Retrospective database } \\
\text { review }\end{array}$ & South Korea & 102 & $\begin{array}{l}\text { All patients }=64 \\
(\text { Mean) }\end{array}$ & 100 \\
\hline Amat-Santos et al. [79] & $\begin{array}{l}\text { Randomized controlled } \\
\text { trial }\end{array}$ & Spain & 11 & All patients $=86$ & 54.5 \\
\hline López-Otero et al. [80] & $\begin{array}{l}\text { Retrospective, single } \\
\text { centre }\end{array}$ & A Coruña, Spain & 965 & $\begin{array}{l}\text { All patients }=60 \\
(\text { Mean) }\end{array}$ & 30.9 \\
\hline Selçuk et al. [81] & Retrospective, multicentre & Istanbul, Turkey & 113 & $\begin{array}{l}\text { ACEI/ARB users }=67 \\
\text { Non-ACEI/ARB } \\
\text { users }=58 \\
\text { (Mean) }\end{array}$ & 100 \\
\hline Baker et al. ${ }^{\mathrm{c}}[82]$ & $\begin{array}{l}\text { Retrospective, single } \\
\text { centre }\end{array}$ & Newcastle, UK & 311 & All patients $=75$ & 42.1 \\
\hline Argenziano et al. [83] & $\begin{array}{l}\text { Retrospective database } \\
\text { review }\end{array}$ & New York, USA & 1000 & All patients $=63$ & 60.1 \\
\hline Chang et al. [84] & Retrospective, multicentre & Los Angeles, USA & 177 & $\begin{array}{l}\text { All patients }=62 \\
\text { (Mean) }\end{array}$ & N/A \\
\hline Chaudhri et al. [85] & $\begin{array}{l}\text { Retrospective, single } \\
\text { centre }\end{array}$ & New York, USA & 300 & $\begin{array}{l}\text { ACEI/ARB users }=56 \\
\text { Non-ACEI/ARB } \\
\text { users }=69 \\
\text { (Mean) }\end{array}$ & 44.3 \\
\hline Imam et al. [86] & Retrospective, multicentre & Michigan, USA & 1305 & $\begin{array}{l}\text { All patients }=61 \\
\text { (Mean) }\end{array}$ & 56.2 \\
\hline Ip et al..$^{c}[87]$ & Retrospective, multicentre & New Jersey, USA & 1129 & - & 100 \\
\hline Khera et al. [88] & $\begin{array}{l}\text { Retrospective database } \\
\text { review }\end{array}$ & USA & 7933 & $\begin{array}{c}\text { ACEI users }=76 \\
\text { ARB users }=76 \\
\text { Non-ACEI/ARB } \\
\text { users }=78\end{array}$ & 100 \\
\hline Lam et al. [89] & $\begin{array}{l}\text { Retrospective, single } \\
\text { centre }\end{array}$ & New York, USA & 335 & All patients $=68$ & 100 \\
\hline Mehta et al. [90] & $\begin{array}{l}\text { Retrospective database } \\
\text { review }\end{array}$ & Ohio and Florida, USA & 1735 & $\begin{array}{l}\text { ACEI/ARB users }=63 \\
\text { Non-ACEI/ARB } \\
\text { users }=65 \\
\text { (Mean) }\end{array}$ & 73.9 \\
\hline Richardson et al. [91] & $\begin{array}{l}\text { Retrospective database } \\
\text { review }\end{array}$ & New York, USA & 1366 & All patients $=63$ & 100 \\
\hline Shah et al. [92] & Retrospective, multicentre & Georgia, USA & 531 & $\begin{array}{l}\text { ACEI/ARB users }=64 \\
\text { Non-ACEI/ARB } \\
\text { users }=58(\text { Mean })\end{array}$ & 80.0 \\
\hline Jung C et al. [93] & Prospective, multicentre & 38 countries & 324 & All patients $=75$ & 65.1 \\
\hline
\end{tabular}

$A C E I$ angiotensin-converting enzyme inhibitor, $A R B$ angiotensin receptor blocker, COVID-19 coronavirus disease 2019, N/A not available ${ }^{\text {a }}$ The total number of COVID-19 patients included in the analysis of mortality and/or severity of disease with the use of ACEIs/ARBs

${ }^{\mathrm{b}}$ Median age unless otherwise stated

${ }^{\mathrm{c}}$ Preprint

compared to the non-use of an ACEI/ARB (Supplementary Figure $\mathrm{S} 1$; pooled $\mathrm{HR}=0.75,95 \%$ CI $0.60-0.95$ ). The funnel plot was used to detect the publication bias and revealed some degree of asymmetry (Supplementary Figure S3 and S4).

Subgroup analysis that was limited to studies that provided adjusted mortality estimates for exclusively hypertensive patients with COVID-19 demonstrated a statistically non-significant association with lower odds of mortality (Supplementary Table S2; pooled OR $=0.73,95 \%$ CI 0.52-1.02; six studies [66, $75,78,88,89,92])$ and a statistically significant association with lower risk of mortality (Supplementary Table S2; pooled $\mathrm{HR}=0.39,95 \%$ CI $0.20-0.77$; five studies $[36,54,56,57,72]$ ) among users of ACEIs/ARBs compared to the non-users. 
Table 2 Comparison of mortality and severe/critical illness between COVID-19 ACEI/ARB users and non-ACEI/ARB users

\begin{tabular}{|c|c|c|c|c|c|c|}
\hline \multirow[t]{2}{*}{ Study } & \multicolumn{3}{|l|}{ Mortality } & \multicolumn{3}{|c|}{ Severe/critical illness ${ }^{\mathrm{a}}$} \\
\hline & $\begin{array}{l}\text { ACEI/ARB users } \\
(n / N ; \%)\end{array}$ & $\begin{array}{l}\text { Non-ACEI/ARB } \\
\text { users }(n / N ; \%)\end{array}$ & $\begin{array}{l}\text { Adjusted esti- } \\
\text { mate }(95 \% \mathrm{CI})\end{array}$ & $\begin{array}{l}\text { ACEI/ARB } \\
\text { users }(n / N ; \\
\%)\end{array}$ & $\begin{array}{l}\text { Non-ACEI/ARB } \\
\text { users }(n / N ; \%)\end{array}$ & $\begin{array}{l}\text { Adjusted esti- } \\
\text { mate }(95 \% \mathrm{CI})\end{array}$ \\
\hline $\begin{array}{l}\text { Amaouche et al. } \\
\text { [35] }\end{array}$ & N/A & N/A & $\begin{array}{l}\mathrm{OR}=1.10 \\
\quad(0.49-2.48)\end{array}$ & N/A & N/A & $\begin{array}{l}\mathrm{OR}=1.12(0.59- \\
2.13)\end{array}$ \\
\hline Chen C et al. [36] & $12 / 355 ; 3.4$ & $95 / 827 ; 11.5$ & $\begin{array}{l}\mathrm{HR}=0.28 \\
\quad(0.15-0.52)\end{array}$ & $6 / 355 ; 1.7$ & $25 / 827 ; 3.0$ & - \\
\hline Chen $\mathrm{M}$ et al. ${ }^{\mathrm{b}}[37]$ & $3 / 11 ; 27.3$ & $28 / 112 ; 25.0$ & - & - & - & - \\
\hline Chen Y et al. [38] & $4 / 32 ; 12.5$ & $10 / 39 ; 25.6$ & - & - & - & - \\
\hline Feng Y et al. [39] & - & - & - & $4 / 33 ; 12.1$ & $36 / 80 ; 45.0$ & - \\
\hline Feng $\mathrm{Z}$ et al. ${ }^{\mathrm{b}}[40]$ & - & - & - & $1 / 16 ; 6.3$ & $16 / 49 ; 32.7$ & $\begin{array}{l}\mathrm{OR}=0.41(0.05- \\
3.19)\end{array}$ \\
\hline Gao et al. [41] & $4 / 183 ; 2.2$ & $\begin{array}{l}\text { Overall cohort: } \\
52 / 2694 ; 1.9 \\
\text { Hypertensive } \\
\text { cohort: } \\
\text { 30/667; } 4.5\end{array}$ & - & $74 / 183 ; 40.4$ & $\begin{array}{l}\text { Overall cohort: } \\
670 / 2694 ; 24.9 \\
\text { Hypertensive } \\
\text { cohort: } \\
221 / 667 ; 33.1\end{array}$ & - \\
\hline Guo et al. [42] & $7 / 19 ; 36.8$ & $43 / 168 ; 25.6$ & - & - & - & - \\
\hline Hu et al. [43] & $1 / 65 ; 1.5$ & $0 / 84 ; 0$ & - & $28 / 65 ; 43.1$ & $33 / 84 ; 39.3$ & - \\
\hline Huang et al. [44] & $0 / 20 ; 0.0$ & $3 / 30 ; 10.0$ & - & $13 / 20 ; 65.0$ & $24 / 30 ; 80.0$ & - \\
\hline Li J et al. [45] & $21 / 115 ; 18.3$ & $56 / 247 ; 22.7$ & - & $57 / 115 ; 49.6$ & $116 / 247 ; 47.0$ & - \\
\hline Li X et al. [46] & - & - & - & $19 / 42 ; 45.2$ & $250 / 506 ; 49.4$ & - \\
\hline Liu et al. ${ }^{\mathrm{b}}[47]$ & - & - & - & $7 / 22 ; 31.8$ & $31 / 56 ; 55.4$ & - \\
\hline Meng et al. [48] & $0 / 17 ; 0.0$ & $1 / 25 ; 4.0$ & - & $4 / 17 ; 23.5$ & $12 / 25 ; 48.0$ & - \\
\hline Peng et al. [49] & $4 / 22 ; 18.2$ & $13 / 90 ; 14.4$ & - & $3 / 22 ; 13.6$ & $13 / 90 ; 14.4$ & - \\
\hline Tan et al. [50] & $0 / 31 ; 0$ & $11 / 69 ; 15.9$ & - & $0 / 31 ; 0$ & $9 / 69 ; 13.0$ & - \\
\hline $\mathrm{Xu}$ et al. [51] & $11 / 40 ; 27.5$ & $21 / 61 ; 34.4$ & - & $8 / 40 ; 20.0$ & $17 / 61 ; 27.9$ & - \\
\hline Yan et al. ${ }^{\text {b }}$ [52] & - & - & - & N/A & N/A & $\begin{array}{l}\text { ACEI: } \\
\text { OR }=1.23(0.19- \\
7.93) \\
\text { ARB: } \\
\text { OR }=0.77(0.36- \\
1.63)\end{array}$ \\
\hline Yang et al. [53] & $2 / 43 ; 4.7$ & $\begin{array}{l}\text { Overall cohort: } \\
\text { 19/208; } 9.1 \\
\text { Hypertensive } \\
\text { cohort: } \\
11 / 83 ; 13.3\end{array}$ & - & $15 / 43 ; 34.9$ & $\begin{array}{l}\text { Overall cohort: } \\
33 / 208 ; 15.9 \\
\text { Hypertensive } \\
\text { cohort: } \\
19 / 83 ; 22.9\end{array}$ & - \\
\hline Yuan et al. [54] & $6 / 130 ; 4.6$ & $22 / 130 ; 16.9$ & $\begin{array}{l}\mathrm{HR}=0.23 \\
\quad(0.09-0.56)\end{array}$ & - & - & - \\
\hline Zeng et al. ${ }^{b}[55]$ & $2 / 28 ; 7.1$ & $\begin{array}{l}\text { Overall cohort: } \\
\text { 19/246; } 7.7 \\
\text { Hypertensive } \\
\text { cohort: } \\
\text { 5/47; } 10.6\end{array}$ & - & $15 / 28 ; 53.6$ & $\begin{array}{l}\text { Overall cohort: } \\
\text { 102/246; } 41.5 \\
\text { Hypertensive } \\
\text { cohort: } \\
\text { 15/47; } 31.9\end{array}$ & - \\
\hline Zhang et al. [56] & $7 / 188 ; 3.7$ & $92 / 940 ; 9.8$ & $\begin{array}{l}\mathrm{HR}=0.37 \\
(0.15-0.89)\end{array}$ & $6 / 188 ; 3.2$ & $75 / 940 ; 8.0$ & $\begin{array}{c}\mathrm{HR}=0.32(0.13- \\
0.80)\end{array}$ \\
\hline
\end{tabular}


Table 2 (continued)

\begin{tabular}{|c|c|c|c|c|c|c|}
\hline \multirow[t]{2}{*}{ Study } & \multicolumn{3}{|l|}{ Mortality } & \multicolumn{3}{|c|}{ Severe/critical illness ${ }^{\mathrm{a}}$} \\
\hline & $\begin{array}{l}\text { ACEI/ARB users } \\
(n / N ; \%)\end{array}$ & $\begin{array}{l}\text { Non-ACEI/ARB } \\
\text { users }(n / N ; \%)\end{array}$ & $\begin{array}{l}\text { Adjusted esti- } \\
\text { mate }(95 \% \mathrm{CI})\end{array}$ & $\begin{array}{l}\text { ACEI/ARB } \\
\text { users }(n / N ; \\
\%)\end{array}$ & $\begin{array}{l}\text { Non-ACEI/ARB } \\
\text { users }(n / N ; \%)\end{array}$ & $\begin{array}{l}\text { Adjusted esti- } \\
\text { mate }(95 \% \mathrm{CI})\end{array}$ \\
\hline Zhou F et al. [57] & N/A & N/A & $\begin{array}{l}\text { Overall cohort } \\
(\text { ACEI/ARB): } \\
\text { HR }=0.39 \\
(0.26-0.58) \\
\text { Hypertensive } \\
\text { cohort } \\
(\text { ACEI/ARB): } \\
\text { HR }=0.32 \\
(0.15-0.66) \\
\text { ACEI: } \\
\text { HR }=0.49 \\
(0.20-1.20) \\
\text { ARB: } \\
\text { HR }=0.31 \\
(0.18-0.53)\end{array}$ & - & - & - \\
\hline Zhou X et al. [58] & $2 / 15 ; 13.3$ & $5 / 21 ; 23.8$ & - & - & - & - \\
\hline Fosbøl et al. [59] & $181 / 895 ; 20.2$ & $297 / 3585 ; 8.3$ & $\begin{array}{l}\text { ACEI/ARB: } \\
\text { HR }=0.83 \\
(0.67-1.03) \\
\text { ACEI: } \\
\text { HR }=0.98 \\
(0.71-1.35) \\
\text { ARB: } \\
\text { HR }=0.80 \\
(0.60-1.09)\end{array}$ & $203 / 895 ; 22.7$ & $373 / 3585 ; 10.4$ & $\begin{array}{l}\text { ACEI/ARB: } \\
\text { HR }=1.15(0.95- \\
1.41) \\
\text { ACEI: } \\
\text { HR }=1.21(0.91- \\
1.60) \\
\text { ARB: } \\
\text { HR }=1.01(0.78- \\
1.31)\end{array}$ \\
\hline Dauchet et al. ${ }^{\mathrm{b}}[60]$ & - & - & - & $34 / 62 ; 54.8$ & $54 / 125 ; 43.2$ & - \\
\hline Liabeuf et al. [61] & $17 / 96 ; 17.7$ & $30 / 172 ; 17.4$ & - & $35 / 96 ; 36.5$ & $34 / 172 ; 19.8$ & $\begin{array}{l}\mathrm{OR}=2.28(1.17- \\
4.42)\end{array}$ \\
\hline Oussalah et al. [62] & $10 / 43 ; 23.3$ & $9 / 104 ; 8.7$ & - & $20 / 43 ; 46.5$ & $34 / 103 ; 33.0$ & - \\
\hline Zhou J et al. ${ }^{\mathrm{b}}[63]$ & - & - & - & $14 / 184 ; 7.6$ & $4 / 792 ; 0.5$ & $\begin{array}{l}\mathrm{OR}=1.10(0.24- \\
2.14)\end{array}$ \\
\hline Cannata et al. [64] & $7 / 56 ; 12.5$ & $39 / 224 ; 17.4$ & $\begin{array}{l}\mathrm{OR}=0.05 \\
\quad(0.01-0.54)\end{array}$ & - & - & - \\
\hline $\begin{array}{l}\text { Conversano et al. } \\
\text { [65] }\end{array}$ & $\begin{array}{l}\text { Overall cohort: } \\
21 / 69 ; 30.4 \\
\text { Hypertensive } \\
\text { cohort: } \\
21 / 68 ; 30.9\end{array}$ & $\begin{array}{l}\text { Overall cohort: } \\
21 / 122 ; 17.2 \\
\text { Hypertensive } \\
\text { cohort: } \\
13 / 28 ; 46.4\end{array}$ & - & - & - & - \\
\hline Felice et al. [66] & $15 / 82 ; 18.3$ & $18 / 51 ; 35.3$ & $\begin{array}{l}\mathrm{OR}=0.56 \\
\quad(0.17-0.83)\end{array}$ & $21 / 82 ; 25.6$ & $25 / 51 ; 49.0$ & $\begin{array}{l}\mathrm{OR}=0.25(0.09- \\
0.66)\end{array}$ \\
\hline Ferrante et al. [67] & N/A & N/A & $\begin{aligned} & \mathrm{HR}=0.68 \\
&(0.41-1.14)\end{aligned}$ & - & - & - \\
\hline $\begin{array}{l}\text { Giacomelli et al. } \\
\text { [68] }\end{array}$ & $14 / 31 ; 45.2$ & $34 / 202 ; 16.8$ & - & - & - & - \\
\hline Grasselli et al. [69] & N/A & N/A & $\begin{array}{l}\text { ACEI: } \\
\text { HR }=0.97 \\
\quad(0.69-1.34) \\
\text { ARB: } \\
\text { HR }=1.05 \\
\quad(0.85-1.29)\end{array}$ & - & - & - \\
\hline
\end{tabular}


Table 2 (continued)

\begin{tabular}{|c|c|c|c|c|c|c|}
\hline \multirow[t]{2}{*}{ Study } & \multicolumn{3}{|l|}{ Mortality } & \multicolumn{3}{|c|}{ Severe/critical illness ${ }^{\mathrm{a}}$} \\
\hline & $\begin{array}{l}\text { ACEI/ARB users } \\
(n / N ; \%)\end{array}$ & $\begin{array}{l}\text { Non-ACEI/ARB } \\
\text { users }(n / N ; \%)\end{array}$ & $\begin{array}{l}\text { Adjusted esti- } \\
\text { mate }(95 \% \mathrm{CI})\end{array}$ & $\begin{array}{l}\text { ACEI/ARB } \\
\text { users }(n / N ; \\
\%)\end{array}$ & $\begin{array}{l}\text { Non-ACEI/ARB } \\
\text { users }(n / N ; \%)\end{array}$ & $\begin{array}{l}\text { Adjusted esti- } \\
\text { mate }(95 \% \mathrm{CI})\end{array}$ \\
\hline Rossi et al. [70] & N/A & N/A & $\begin{array}{l}\text { ACEI: } \\
\text { HR = } 1.17 \\
(0.97-1.42) \\
\text { ARB: } \\
\text { HR }=1.16 \\
(0.83-1.64)\end{array}$ & - & - & - \\
\hline Sardu et al. [71] & $7 / 45 ; 15.6$ & $2 / 17 ; 11.8$ & - & $9 / 45 ; 20.0$ & $3 / 17 ; 17.6$ & - \\
\hline Tedeschi et al. [72] & N/A & N/A & $\begin{array}{l}\mathrm{HR}=0.97 \\
\quad(0.68-1.39)\end{array}$ & - & - & - \\
\hline Ayed et al. [73] & $5 / 10 ; 50.0$ & $10 / 93 ; 10.8$ & - & - & - & - \\
\hline Dalan et al. [74] & N/A & N/A & $\begin{array}{l}\text { ACEI: } \\
\text { HR }=0.50 \\
(0.08-3.24) \\
\text { ARB: } \\
\text { HR }=2.87 \\
(0.41-20.00)\end{array}$ & N/A & N/A & $\begin{array}{l}\text { ACEI: } \\
\text { HR }=0.26(0.10- \\
0.68) \\
\text { ARB: } \\
\text { HR }=2.19(1.08- \\
4.43)\end{array}$ \\
\hline Choi et al. [75] & $42 / 625 ; 6.7$ & $69 / 625 ; 11.0$ & $\begin{array}{l}\mathrm{OR}=0.48 \\
\quad(0.29-0.79)\end{array}$ & $34 / 625 ; 5.4$ & $55 / 625 ; 8.8$ & $\begin{array}{l}\mathrm{OR}=0.49(0.30- \\
0.79)\end{array}$ \\
\hline Jung $S$ et al. [76] & $33 / 377 ; 8.8$ & $51 / 1577 ; 3.2$ & $\begin{array}{l}\mathrm{OR}=0.88 \\
\quad(0.53-1.44)\end{array}$ & - & - & - \\
\hline Lee et al. ${ }^{\mathrm{b}}[77]$ & $50 / 977 ; 5.1$ & $62 / 7289 ; 0.85$ & $\begin{array}{l}\mathrm{HR}=1.07 \\
\quad(0.68-1.65)\end{array}$ & - & - & - \\
\hline Son et al. [78] & $30 / 77 ; 39.0$ & $8 / 25 ; 32.0$ & $\begin{array}{l}\text { ACEI/ARB: } \\
\text { OR }=1.36(0.51- \\
3.66) \text { ACEI: } \\
\text { OR }=0.26 \\
(0.03-2.26) \\
\text { ARB: } \\
\text { OR }=2.10 \\
(0.83-5.34)\end{array}$ & $18 / 51 ; 35.3$ & $4 / 15 ; 26.7$ & $\begin{array}{l}\text { ACEI/ARB: } \\
\text { OR }=1.52(0.40- \\
5.70) \\
\text { ACEI: } \\
\text { OR }=2.24(0.13- \\
37.88) \\
\text { ARB: } \\
\text { OR }=1.70(0.46- \\
6.32)\end{array}$ \\
\hline $\begin{array}{l}\text { Amat-Santos et al. } \\
\text { [79] }\end{array}$ & $2 / 5 ; 40.0$ & $2 / 6 ; 33.3$ & - & - & - & - \\
\hline $\begin{array}{l}\text { López-Otero et al. } \\
\text { [80] }\end{array}$ & $11 / 210 ; 5.2$ & $27 / 755 ; 3.6$ & $\begin{array}{l}\text { ACEI/ARB: } \\
\text { OR }=0.62 \\
(0.17-2.26) \\
\text { ACEI: } \\
\text { OR }=0.14 \\
(0.01-1.57) \\
\text { ARB: } \\
\text { OR }=1.54 \\
(0.42-5.59)\end{array}$ & $13 / 78 ; 16.7$ & $20 / 156 ; 12.8$ & $\begin{array}{l}\text { ACEI/ARB: } \\
\text { OR }=0.87(0.30- \\
2.50) \\
\text { ACEI: } \\
\text { OR }=0.97(0.22- \\
4.16) \\
\text { ARB: } \\
\text { OR }=0.84(0.25- \\
2.87)\end{array}$ \\
\hline Selçuk et al. [81] & $31 / 74 ; 41.9$ & $4 / 39 ; 10.3$ & $\begin{array}{l}\mathrm{OR}=3.66 \\
\quad(1.11-18.18)\end{array}$ & - & - & - \\
\hline Baker et al. ${ }^{\mathrm{b}}[82]$ & $17 / 78 ; 21.8$ & $63 / 233 ; 27.0$ & - & - & - & - \\
\hline $\begin{array}{l}\text { Argenziano et al. } \\
\text { [83] }\end{array}$ & - & - & - & $71 / 284 ; 25.0$ & $165 / 716 ; 23.0$ & - \\
\hline Chang et al. [84] & - & - & - & $6 / 27 ; 22.2$ & $50 / 150 ; 33.3$ & $\begin{array}{l}\text { ACEI: } \\
\text { OR }=0.62(0.14- \\
2.20) \\
\text { ARB: } \\
\text { OR }=0.50(0.11- \\
1.80)\end{array}$ \\
\hline Chaudhri et al. [85] & $14 / 80 ; 17.5$ & $25 / 220 ; 11.4$ & - & $22 / 80 ; 27.5$ & $59 / 220 ; 26.8$ & - \\
\hline
\end{tabular}


Table 2 (continued)

\begin{tabular}{|c|c|c|c|c|c|c|}
\hline \multirow[t]{2}{*}{ Study } & \multicolumn{3}{|l|}{ Mortality } & \multicolumn{3}{|c|}{ Severe/critical illness ${ }^{\mathrm{a}}$} \\
\hline & $\begin{array}{l}\text { ACEI/ARB users } \\
(n / N ; \%)\end{array}$ & $\begin{array}{l}\text { Non-ACEI/ARB } \\
\text { users }(n / N ; \%)\end{array}$ & $\begin{array}{l}\text { Adjusted esti- } \\
\text { mate }(95 \% \mathrm{CI})\end{array}$ & $\begin{array}{l}\text { ACEI/ARB } \\
\text { users }(n / N ; \\
\%)\end{array}$ & $\begin{array}{l}\text { Non-ACEI/ARB } \\
\text { users }(n / N ; \%)\end{array}$ & $\begin{array}{l}\text { Adjusted esti- } \\
\text { mate }(95 \% \mathrm{CI})\end{array}$ \\
\hline Imam et al. [86] & N/A & N/A & $\begin{array}{l}\mathrm{OR}=1.20 \\
(0.86-1.68)\end{array}$ & - & - & - \\
\hline Ip et al. ${ }^{\mathrm{b}}[87]$ & $137 / 460 ; 29.8$ & $262 / 669 ; 39.2$ & - & - & - & - \\
\hline Khera et al. [88] & $664 / 4587 ; 14.5$ & $466 / 3346 ; 13.9$ & $\begin{array}{l}\text { ACEI: } \\
\text { OR }=0.97 \\
(0.81-1.16) \\
\text { ARB: } \\
\text { OR }=1.15 \\
(0.95-1.38)\end{array}$ & - & - & - \\
\hline Lam et al. [89] & $10 / 164 ; 6.1$ & $48 / 171 ; 28.1$ & $\begin{array}{l}\mathrm{OR}=0.22 \\
\quad(0.10-0.46)\end{array}$ & $20 / 164 ; 12.2$ & $45 / 171 ; 26.3$ & $\begin{array}{c}\mathrm{OR}=0.35(0.19- \\
0.64)\end{array}$ \\
\hline Mehta et al. [90] & $8 / 211 ; 3.8$ & $34 / 1494 ; 2.3$ & - & $22 / 212 ; 10.4$ & $15 / 1523 ; 0.98$ & $\begin{array}{l}\text { ACEI/ARB: } \\
\text { OR }=1.64(1.07- \\
2.51) \\
\text { ACEI: } \\
\text { OR }=1.77(1.07- \\
2.92) \\
\text { ARB: } \\
\text { OR }=1.16(0.67- \\
2.02)\end{array}$ \\
\hline $\begin{array}{l}\text { Richardson et al. } \\
\text { [91] }\end{array}$ & $130 / 413 ; 31.5$ & $254 / 953 ; 26.7$ & - & $87 / 413 ; 21.1$ & $141 / 953 ; 14.8$ & - \\
\hline Shah et al. [92] & $38 / 207 ; 18.4$ & $48 / 324 ; 14.8$ & $\begin{array}{l}\text { Overall cohort: } \\
\text { OR }=0.82 \\
(0.45-1.50) \\
\text { Hypertensive } \\
\text { cohort: } \\
\text { OR }=0.79 \\
(0.43-1.44)\end{array}$ & $59 / 207 ; 28.5$ & $62 / 324 ; 19.1$ & $\begin{array}{l}\text { Overall cohort: } \\
\text { OR }=1.26(0.74- \\
2.15) \\
\text { Hypertensive cohort: } \\
\text { OR }=1.25(0.72- \\
2.16)\end{array}$ \\
\hline Jung C et al. [93] & $62 / 157 ; 39.5$ & $85 / 167 ; 50.9$ & $\begin{array}{l}\text { ACEI: } \\
\text { OR }=0.32 \\
\quad(0.15-0.67)\end{array}$ & - & - & - \\
\hline
\end{tabular}

$A C E I$ angiotensin-converting enzyme inhibitor, $A R B$ angiotensin receptor blocker, $C I$ confidence interval, COVID-19 coronavirus disease 2019 , $H R$ hazard ratio, N/A not available, $O R$ odds ratio

${ }^{a}$ The definition of severe/critical disease in the studies by Amaouche et al. [35], Feng et al. [39], Feng et al. [40], Gao et al. [41], Hu et al. [43], Huang et al. [44], Li et al. [45], Li et al. [46], Liu et al. [47], Meng et al. [48], Peng et al. [49], Yan et al. [52], and Yang et al. [53] is based on the definition given in 'Diagnosis and Treatment Protocol for Novel Coronavirus Pneumonia' by Chinese National Health Commission; Chen et al. [36] is based on the development of multiple organ dysfunction syndrome; Tan et al. [50] is based on the development of acute respiratory distress syndrome; Xu et al. [51], Fosbøl et al. [59], Dauchet et al. [60], Liabeuf et al. [61], Zhou et al. [63], Felice et al. [66], Sardu et al. [71], Dalan et al. [74], Son et al. [78], López-Otero et al. [80], Argenziano et al. [83], Chaudhri et al. [85], Lam et al. [89], Mehta et al. [90], Richardson et al. [91], and Shah et al. [92] are based on the requirement for admission into intensive care units; Zeng et al. [55] is based on the guideline for community-acquired pneumonia; Zhang et al. [56] is based on the development of septic shock; Oussalah et al. [62] is based on the requirement for intubation or mechanical ventilation; Choi et al. [75] is based on the requirement for mechanical ventilation, admission into intensive care units, continuous renal replacement therapy, or extracorporeal membrane oxygenation; and Chang et al. [84] is based on the requirement for admission into intensive care units or intubation

${ }^{\mathrm{b}}$ Preprint

Subgroup analysis based on the region where the studies were performed demonstrated a non-significant association with lower odds of mortality in studies originated from East Asia (Supplementary Table S2; pooled OR $=0.76,95 \%$ CI 0.44-1.31) [75, 76, 78], Europe (Supplementary Table S2; pooled OR $=0.51,95 \%$ CI $0.21-1.25)$ [35, 64, 66, 80], and the United States (Supplementary Table S2; pooled $\mathrm{OR}=0.89,95 \% \mathrm{CI} 0.66-1.21)[86,88,89,92]$ in the users of ACEIs/ARBs compared to the non-users.

Subgroup analyses that were limited to studies that provided respective mortality estimates (adjusted OR) for ACEI and ARB showed a statistically non-significant association 
Fig. 2 Pooled mortality estimate (OR) associated with the use of ACEIs/ARBs. Heterogeneity: $I^{2}=74 \% ; p=0.001$. ACEI angiotensin-converting enzyme inhibitor, $A R B$ angiotensin receptor blocker, $C I$ confidence interval, $O R$ odds ratio

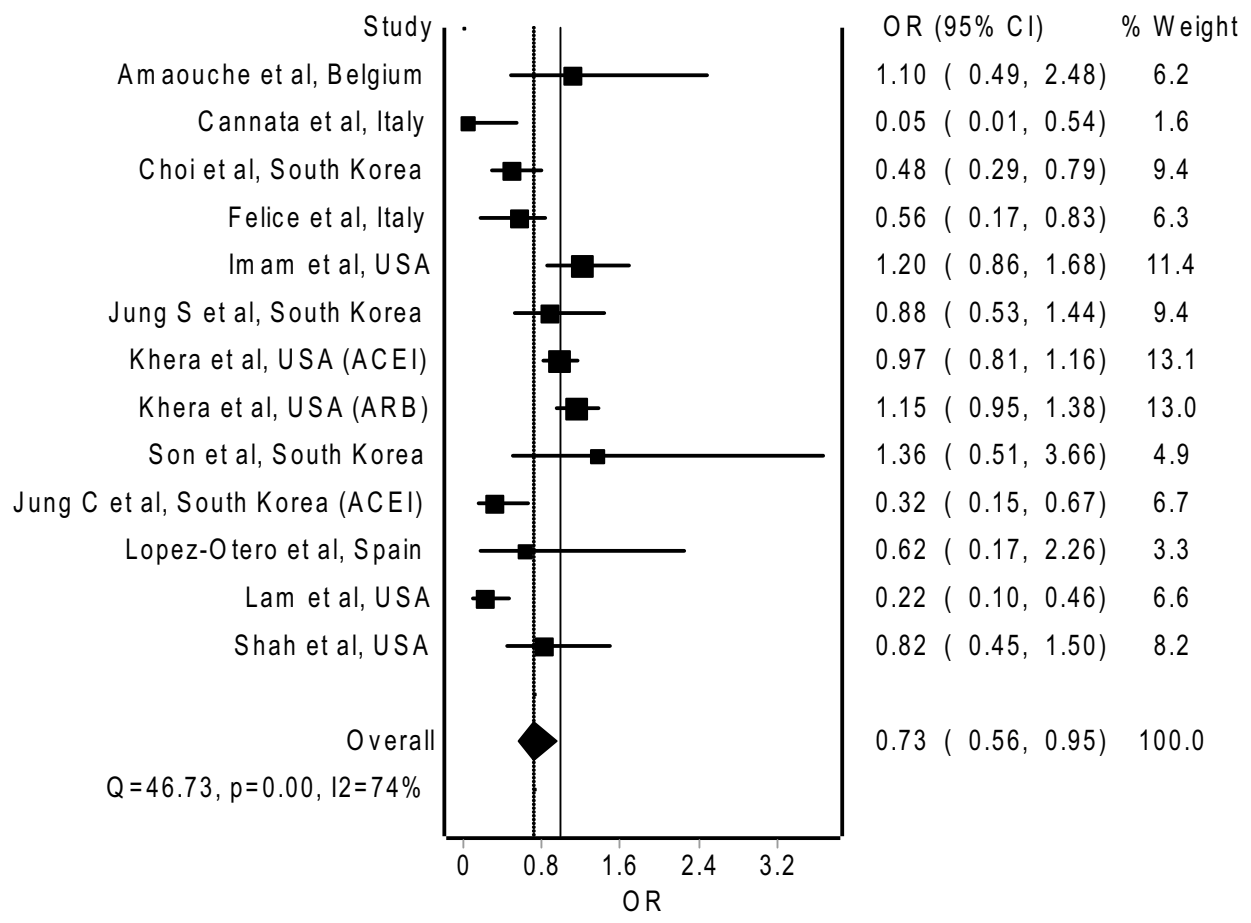

with lower odds of mortality with the use of an ACEI compared to non-use of an ACEI (Supplementary Table S2; pooled $\mathrm{OR}=0.46,95 \%$ CI $0.18-1.17$; four studies $[78,80$, $88,93])$, but higher odds of mortality with the use of an ARB (Supplementary Table S2; pooled OR $=1.18,95 \%$ CI $0.99-1.42$; three studies $[78,80,88]$ ) compared to non-use of an ARB, among COVID-19 patients.

Subgroup analyses in the studies that provided respective mortality estimates (adjusted HR) for ACEIs and ARBs showed a statistically non-significant association with higher risk of mortality with the use of an ACEI (Supplementary Table S2; pooled HR $=1.03$, 95\% CI 0.85-1.23; five studies $[57,59,69,70,74])$, but lower risk of mortality with the use of an ARB (Supplementary Table S2; pooled HR $=0.82$, 95\% CI 0.55-1.24; five studies [57, 59, 69, 70, 74]) compared to non-use of an ACEI and an ARB, respectively, among COVID-19 patients.

\subsection{Severe/Critical Outcomes Associated with the Use of RAS Inhibitors in COVID-19 Patients}

There were 16 studies [35, 40, 52, 56, 59, 61, 63, 66, 74, $75,78,80,84,89,90,92]$ that provided adjusted estimates for severe/critical disease with the use of an ACEI/ARB relative to the non-use of an ACEI/ARB. Non-uniformity in the definition of the severe/critical outcomes was observed amongst the 16 included studies. Three studies [35, 40, 52] defined severe/critical cases of COVID-19 according to the 'Diagnosis and Treatment Protocol for Novel Coronavirus Pneumonia' by the Chinese National Health Commission. Choi et al. [75] defined severe/critical outcomes from COVID-19 based on the requirement for mechanical ventilation, admission into intensive care units, continuous renal replacement therapy, or extracorporeal membrane oxygenation; whilst Chang et al. [84] defined severe/critical outcomes from COVID-19 based on the requirement for admission into intensive care units or intubation. Eleven studies did not classify patients into severe/critical cohort and non-severe/critical cohort; however, in our analyses, we considered patients with septic shock (for Zhang et al. [56]) and those admitted into intensive care units (for the remaining ten studies $[59,61,63,66,74,78,80,89,90,92])$ as having a severe/critical course of COVID-19.

The Newcastle-Ottawa scale [95] was used for the quality assessment of all studies that provided adjusted estimates and were included in the meta-analysis of severe/critical outcomes associated with the use of ACEIs/ARBs (Supplementary Table S1). Of the 16 original studies included, only one study, by Zhang et al. [56], was deemed 'good', with a score of 7 points. Ten studies [35, 40, 61, 63, 66, 75, 78, 89, $90,92]$ were regarded as 'fair', with scores of 4-6 points. Five studies [52, 59, 74, 80, 84] were considered 'poor' (i.e., scored less than 4 points) in the quality assessment.

The main quality issue noticed in 14 studies [35, 40, 52, $59,61,63,66,74,78,80,84,89,90,92]$ was the comparison of cohorts with inadequate adjustment for confounders that may influence the estimated risk of severe/critical 
disease associated with the use of ACEIs/ARBs. Another major issue detected during quality assessment in 12 studies $[35,52,59,61,66,74,75,78,80,84,90,92]$ was the inability to ascertain the exposure to ACEIs/ARBs during the course of illness, where the possibility of discontinuation of ACEIs/ARBs upon COVID-19 diagnosis could not be ruled out based on the study design. Similarly, 12 studies $[40,52,59,61,63,74,75,78,84,89,90,92]$ were not able to demonstrate that severe/critical outcome was not present at the start of the study based on their study design. In 11 studies $[35,52,59,63,66,74,75,78,80,84,90]$, it was unclear whether the entire cohort of patients was followed until discharge/death. The representativeness of the exposed cohort could not be established in ten studies [40, 52, 56, $59,63,74,80,84,89,92]$ that included hospitalized patients only.

In a pooled analysis of 13 original studies that provided adjusted ORs, with 7446 COVID-19 patients being analyzed $[35,40,52,61,63,66,75,78,80,84,89,90,92]$, the use of an ACEI/ARB was non-significantly associated with lower odds of developing severe/critical disease compared to the non-use of an ACEI/ARB (Fig. 3; pooled OR $=0.91,95 \%$ CI 0.75-1.10). In a separate pooled analysis of three studies that provided adjusted HRs, with 6325 COVID-19 patients being analyzed [56, 59, 74], the use of an ACEI/ARB was non-significantly associated with a lower risk of developing severe/critical disease compared to the non-use of an ACEI/ ARB (Supplementary Figure S2; pooled HR $=0.73,95 \%$ CI
0.33-1.66). The funnel plot was used to detect the publication bias and revealed some degree of asymmetry.

Subgroup analysis that was limited to six studies that provided adjusted mortality estimates for exclusively hypertensive patients with COVID-19 [52, 66, 75, 78, 89, 92] demonstrated a statistically significant association with lower odds of developing severe/critical disease (Supplementary Table S2; pooled OR $=0.63,95 \%$ CI 0.41-0.96). Subgroup analyses based on the region where the studies were performed demonstrated a statistically significant association with lower odds of developing severe/critical disease from studies originated from East Asian countries (Supplementary Table S2; pooled OR $=0.70,95 \%$ CI $0.52-0.93$ ) [40, $52,63,75,78]$, but a statistically non-significant association with higher odds of developing severe/critical disease from studies originated from European countries (Supplementary Table S2; pooled OR $=1.02,95 \%$ CI $0.61-1.70)[35,61$, $66,80]$ and lower odds of developing severe/critical disease from studies originated from the United States (Supplementary Table S2; pooled OR $=0.80,95 \%$ CI $0.40-1.61$ ) [84, 89, 90, 92], among users of ACEIs/ARBs compared to non-users.

Subgroup analyses limited to the studies that provided respective estimates for severe/critical outcomes (adjusted OR) for ACEIs and ARBs observed a statistically significant association with higher odds of development of severe/ critical illness with the use of an ACEI (Supplementary Table S2; pooled OR $=1.50,95 \%$ CI 1.04-2.14; five studies
Fig. 3 Pooled estimate (OR) of severe/critical illness associated with the use of ACEIs/ ARBs. Heterogeneity: $I^{2}=66 \%$; $p=0.001$. ACEI angiotensinconverting enzyme inhibitor, $A R B$ angiotensin receptor blocker, $C I$ confidence interval, $O R$ odds ratio

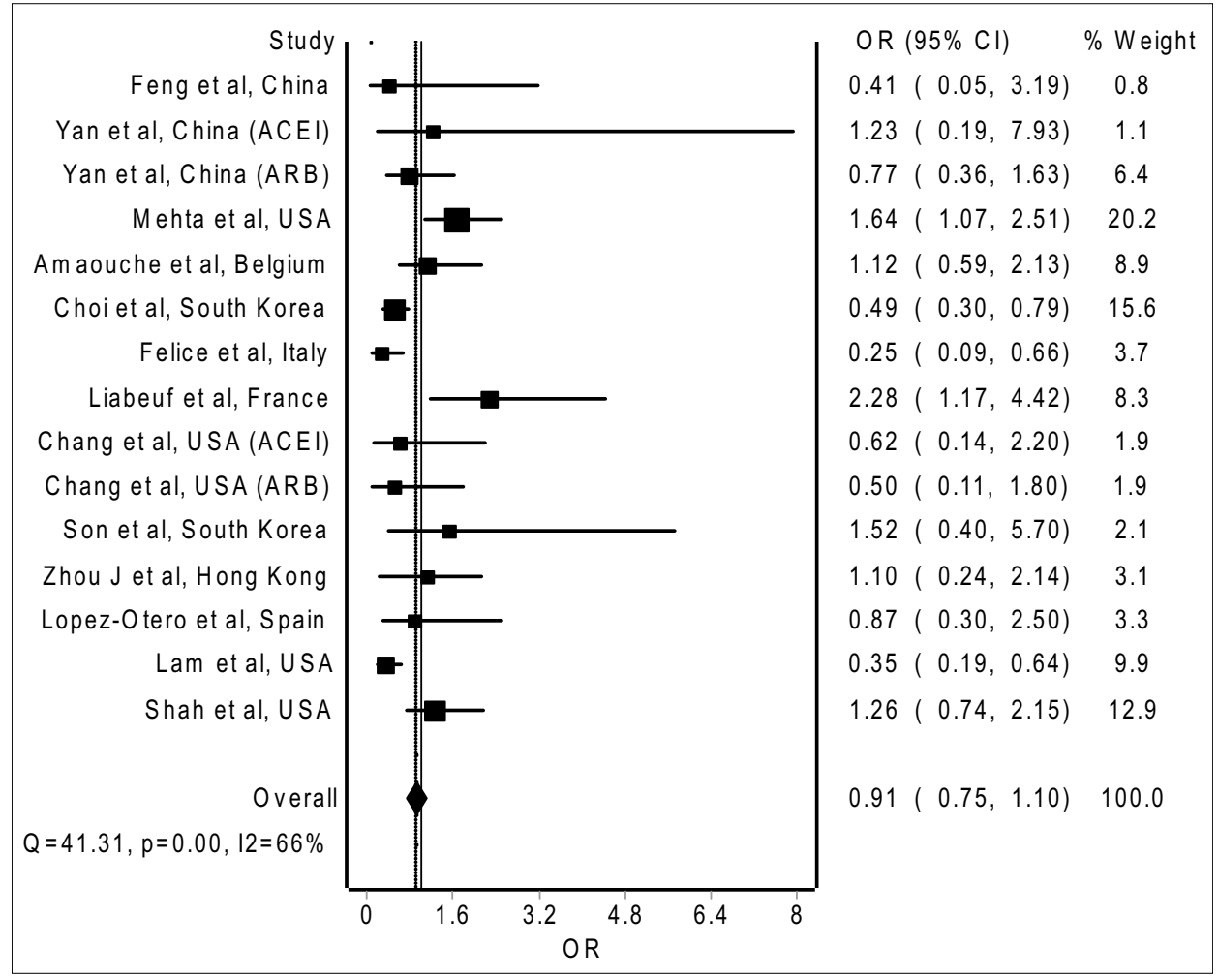


$[52,78,80,84,90])$, but a statistically non-significant association with lower odds of development of severe/critical illness with the use of an ARB (Supplementary Table S2; pooled OR $=0.98,95 \%$ CI 0.67-1.44; five studies [52, 78, $80,84,90])$, compared to the non-use of an ACEI and an ARB, respectively, among patients with COVID-19.

Subgroup analysis with regards to different definitions of severe/critical outcomes used across the studies reported a statistically non-significant association with lower odds of developing severe/critical illness according to the definitions of the Chinese National Health Commission (Supplementary Table S2; pooled OR $=0.93,95 \%$ CI 0.59-1.48; three studies [35, 40, 52]) and a non-significant association with lower odds of being admitted into intensive care units (Supplementary Table S2; pooled OR $=0.91,95 \%$ CI 0.56-1.49; eight studies $[61,63,66,78,80,89,90,92])$, with the use of ACEIs/ARBs compared to the non-use of ACEIs/ARBs.

\section{Discussion}

To the best of our knowledge, this systematic review and meta-analysis is the most comprehensive exploration and analysis of the existing literature in terms of mortality and clinical outcomes with the use of RAS inhibitors to date. An in-depth analysis of study methods and findings for each original study is of utmost importance to prevent false assumptions about the safety of RAS inhibitors in COVID19. From our literature review, there have been a large number of observational studies [35-38, 41-45, 48-51, 53-59, $61,62,64-82,85-93$ ] thus far evaluating the association between the use of ACEIs/ARBs in COVID-19 and mortality since concerns were raised with regard to the potential harms from the use of these drugs. However, conflicting results were observed among these studies. There were 23 studies [37, 41-43, 49, 59, 61, 62, 65, 68, 71, 73, 76-81, 85, $88,90-92]$ that reported a higher crude mortality rate; conversely 19 studies $[36,38,44,45,48,50,51,53-56,58,64$, $66,75,82,87,89,93$ ] reported a lower crude mortality rate among ACEI/ARB users with COVID-19 compared with their counterparts without receiving ACEIs/ARBs. However, as mentioned earlier, there were only a few studies that adjusted covariates that may confound the association between the use of ACEIs/ARBs in COVID-19 and the risk of mortality. With different extents of adjustment for covariables, all [59, 76-78, 80, 88, 92] but one study [81] (which reported a higher crude mortality rate among ACEI/ARB users compared to non-ACEI/ARB users) demonstrated no significant difference in the risk of mortality between ACEI/ ARB users and non-ACEI/ARB. The study by Selçuk et al. [81] reported a higher crude mortality rate and provided mortality estimates with a very wide CI $(\mathrm{OR}=3.66 ; 95 \%$ CI 1.11-18.18); therefore, the reliability of their findings is questionable. In fact, in our random-effects meta-analysis of studies that provided adjusted mortality estimates, we observed significantly lower risk of mortality with the use of ACEIs/ARBs in COVID-19 patients relative to non-use of ACEIs/ARBs. Our findings are in line with previously reported meta-analyses [96-100] with unadjusted estimates that demonstrated no increased risk of mortality with the use of ACEIs/ARBs in COVID-19 patients.

Similarly, upon extensive literature review, we found that a vast number of observational studies [35, 36, 39-41, 43-53, 55, 56, 59-63, 66, 71, 74, 75, 78, 80, 83-85, 89-92] have thus far evaluated the association between the use of ACEIs/ARBs in COVID-19 and clinical severity of illness, albeit with conflicting results. There were 18 studies [41, 43, $45,53,55,59-63,71,78,80,83,85,90-92]$ that reported a higher crude rate for the severe/critical outcomes; however, 15 studies [36, 39, 40, 44, 46-51, 56, 66, 75, 84, 89] reported a lower crude rate for severe/critical clinical outcomes, in ACEI/ARB users with COVID-19 compared to non-users. Nonetheless, only a few studies adjusted covariates that may have confounded the association between the use of ACEIs/ ARBs in COVID-19 and clinical severity. With different extents of adjustment for covariables, among seven studies $[59,61,63,78,80,90,92]$ that reported a higher crude rate for severe/critical outcomes, five studies [59, 63, 78, 80, 92] demonstrated no significant difference with regards to the risk of severe/critical outcomes between ACEI/ARB users and non-ACEI/ARB users. Conversely, Liabeuf et al. [61] and Mehta et al. [90] demonstrated significantly higher odds for admission into intensive care units with the use of an ACEI/ARB relative to the non-use of ACEI/ARB upon adjustment of covariates, but arguably this may be due to the residual confounding effect. In fact, the random-effects meta-analysis of studies with adjusted estimates observed no significant association between the use of ACEIs/ARBs and severe/critical outcomes in COVID-19 patients, which concurs with previously reported unadjusted meta-analyses [96, $99,101]$ that showed no increased risk of developing severe/ critical illness with the use of ACEIs/ARBs in COVID-19 patients. Nevertheless, an unadjusted meta-analysis [102] reported a significantly reduced risk of developing severe/ critical illness with the use of ACEIs/ARBs in COVID-19 patients.

The subgroup analysis is important to determine the presence of heterogeneity on the effect of RAS inhibitors based on different definitions of severe/critical outcomes. We observed no significant association with the use of ACEIs/ ARBs and severe/critical outcomes based on the definition by the Chinese National Health Commission. This is in line with another meta-analysis [96] that pooled studies that defined the clinical severity based on the Chinese National Health Commission. We also observed no significant 
association with the use of ACEIs/ARBs and severe/critical outcomes based on admission into intensive care units.

When studies that included hypertensive patients exclusively or provided a separate analysis for hypertensive patients were analyzed, we observed a greater number of studies $(n=17)[36,38,41,44,45,48,50,51,53-56,58$, $66,75,87,89$ ] reporting a lower crude mortality rate than studies $(n=8)$ [43, 65, 71, 78, 81, 88, 91, 92] reporting higher crude mortality rates in COVID-19 hypertensive ACEI/ARB users relative to the non-ACEI/ARB users. With the exception of the study by Selçuk et al. [81], all studies that reported a higher crude mortality rate [78, 88, 92] demonstrated no significant difference with regards to the risk of mortality between ACEI/ARB users and non-ACEI/ ARB users upon adjustment for covariables. We observed a significantly lower risk of mortality in COVID-19 ACEI/ ARB users in our subgroup meta-analyses that were limited to studies that provided adjusted mortality estimates for exclusively hypertensive patients. These findings are in line with two previously reported unadjusted meta-analyses in COVID-19 hypertensive patients [102, 103]. However, another meta-analysis [97] reported no significant association between the use of ACEIs/ARBs and mortality among COVID-19 patients with hypertension where the authors pooled mortality estimates without adjustment of covariates.

Likewise, for studies that included hypertensive patients exclusively or provided a separate analysis for hypertensive patients, we also observed a greater number of studies ( $n=11)$ [36, 39, 44, 47, 48, 50, 51, 56, 66, 75, 89] reporting a lower crude rate for severe/critical outcomes than studies $(n=8)[41,43,45,53,55,71,78,91]$ reporting a higher crude rate for severe/critical outcomes, though the only study [78] that reported a higher crude rate for severe/critical outcomes and provided an adjusted estimate demonstrated no significant difference with regards to the risk of severe/ critical outcomes between ACEI/ARB users and non-ACEI/ ARB users. Indeed, our subgroup meta-analyses that were limited to studies that provided adjusted estimates for severe/ critical outcomes in exclusively hypertensive patients noted a significantly reduced risk of developing severe/critical disease among COVID-19 ACEI/ARB users.

It is interesting to observe that ACEI/ARB users with or without hypertension had a lower risk of death from COVID-19, but no difference with regards to the risk of developing severe/critical illness from COVID-19, compared to non-ACEI/ARB users with or without hypertension. Equally interesting is the observation that hypertensive ACEI/ARB users had a lower risk of death and a lower risk of developing severe/critical disease from COVID-19 compared to hypertensive non-ACEI/ARB users. One explanation for such observations is that RAS inhibitors may be able to protect COVID-19 patients from angiotensin II-related lung injury and subsequent ARDS, the major cause of mortality in COVID-19 patients [104]. However, since the definition of severe/critical illness in our meta-analysis did not specifically include ARDS, such a protective effect was not reflected in the risk of developing severe/critical illness in our analysis. In fact, a retrospective case-control study [105] performed before the COVID-19 pandemic reported better survival rates among patients with ARDS who received RAS inhibitors compared to their counterparts who did not receive RAS inhibitors. Another plausible explanation for the observations is that hypertensive patients may be more sensitive to the protective effects of RAS inhibitors against the development of a fatal or severe/critical course of illness from COVID-19 since they have a higher baseline risk of a worse prognosis. In fact, the mortality benefits demonstrated in the meta-analysis were driven mainly by studies that provided adjusted mortality estimates for exclusively hypertensive patients (constituting 53\% of the weight of the meta-analysis on mortality). In contrast, studies that provided adjusted estimates for severe/critical outcomes in exclusively hypertensive patients only constituted $23.7 \%$ of the weight of the meta-analysis on severe/critical illness.

Since previous meta-analyses [96-102] included a majority of studies from China, the generalizability of their findings to patient populations from other regions was also questioned. In the present study, we observed no regional difference with regard to mortality estimates, where separate pooled analyses of studies from East Asian countries, European countries, and the United States, respectively, did not find an increased risk of mortality with the use of ACEIs/ ARBs. Nonetheless, our findings did suggest a potential regional difference in the risk of severe/critical outcomes with the use of ACEIs/ARBs in which pooled analysis of studies from East Asian countries demonstrated a significant association with lower odds of developing severe/critical disease compared to the studies from Europe and the United States. Since East Asian populations have a genetically higher expression of ACE2 compared to Caucasians (Europeans or Americans) [106], the downregulation of ACE2 upon a viral invasion of the host cells by SARS-CoV-2 may be consequently greater in East Asian populations. Therefore, East Asian populations with COVID-19 may be more sensitive to the protective effect of RAS inhibitors against lung injuries from downregulation of ACE2 and the subsequent development of severe/critical illness, though it does not appear to translate to any significant mortality benefits. Nevertheless, these findings should be confirmed in a large pharmacogenetic study comparing the effects of ACEIs/ ARBs in COVID-19 patients of different ethnicities.

While both ACEIs and ARBs could prevent lung injuries induced by angiotensin II in COVID-19, ARBs can provide further protection due to their different mechanisms of action. In its native state, the angiotensin type I receptor (AT1R) binds to ACE2 to form a receptor complex [107]. 
ARBs stabilize the ACE2-AT1 receptor complex on the cell membrane and thus prevent the interaction of SARS-CoV-2 with the ACE2 catalytic site. In addition, ARBs are reported to possess anti-inflammatory effects due to their ability to utilize the ACE2/angiotensin II type 2 receptor/mas receptor pathway that could limit SARS-CoV-2-mediated cytokine storm. It is therefore interesting to determine the respective effects of ACEIs and ARBs in COVID-19 patients. We demonstrated no significant difference in the risk of mortality with the use of either ACEIs or ARBs in COVID-19 patients compared to non-use of either of these two agents. Surprisingly, we found that ACEIs were associated with higher odds of developing severe/critical illness from COVID-19 relative to non-use of ACEIs, while no difference in the risk of developing severe/critical illness from COVID-19 was found with the use of ARBs relative to non-use of ARBs. In the pooled analysis, the increased risk of developing severe/critical illness from COVID-19 is largely driven by the findings in the study by Mehta et al. [90] (constituting $71 \%$ of the weight of the meta-analysis), which revealed higher odds of admission into intensive care units with the use of ACEIs compared to the non-use of ACEIs. It should be noted that there may be confounding by indication since Mehta et al. [90] compared the clinical outcomes between COVID-19 users of ACEIs who had comorbidities and COVID-19 non-users of ACEIs who may or may not have had comorbidities. In fact, the other studies [52, 78, 80,84] included in the subgroup pooled analysis reported no difference in the risk of severe/ critical illness with the use of ACEIs compared to non-use of the ACEIs.

A key strength of this systematic review and meta-analysis was the pooling of adjusted estimates on the mortality and severe/critical outcomes from the use of RAS inhibitors in COVID-19 patients. Previous meta-analyses were either unadjusted [96-103, 108, 109] or only pooled adjusted estimates for combined mortality and severity endpoints [110]. Inclusion of studies that provided adjusted estimates would reduce the confounding effects that could modify the association between the use of RAS inhibitors and risk of adverse clinical outcomes in COVID-19. Furthermore, independent analysis of the risk of mortality and the risk of severe/critical outcomes, instead of combining mortality and severity endpoints, enabled the evaluation if the effect of RAS inhibitors on the risk of severe/critical outcomes will translate the same to the risk of mortality. As discussed above, we have observed on two occasions that the effect of the use of RAS inhibitors on severe/critical outcomes did not translate the same to the risk of mortality. Another key strength of our systematic review and meta-analysis lies in our comprehensive inclusion of a large number of studies across different countries and continents. Previous metaanalyses included studies mainly from China and therefore were limited regarding the generalizability of their findings to other populations. Inclusion of a large number of studies with adjusted estimates allowed different subgroup analyses to be performed in our study, including subgroup analysis on hypertensive cohort, which reduced confounding by indication, subgroup analysis stratified by the regions where the studies were conducted, to evaluate the regional difference in the effects of RAS inhibitors on clinical outcomes in COVID-19, and subgroup analysis to determine the respective effects of ACEIs and ARBs on clinical outcomes in COVID-19.

Nonetheless, this study has also limitations that should be considered while interpreting the findings. Notably, although we pooled only studies that provided adjusted estimates, most of the studies did not adequately adjust for all confounders. Other than demographic characteristics, major confounders include comorbidities, co-medications, and the therapies intended for treating COVID-19. It is acknowledged that patients with comorbidity, especially cardiovascular diseases and diabetes mellitus, are particularly vulnerable to the severe course of COVID-19 [111, 112]. Moreover, some co-medications such as statins are reported to exert protective effects on mortality or the development of severe/ critical disease from COVID-19 [113, 114]. In addition, the varying proportion of patients receiving various potential therapies for COVID-19 in the absence of an established treatment may also confound the association between the use of RAS inhibitors and clinical outcomes from COVID19. Among the studies included in our meta-analysis, only two studies properly adjusted for major confounders (Zhang et al. [56] and Zhou et al. [57]), and coincidentally these two studies also reported a significantly reduced risk of mortality from COVID-19 with the use of RAS inhibitors. Despite these findings, residual confounding cannot be completely ruled out, like in any observational study. Moreover, limited by the study designs, we could not establish with certainty whether RAS inhibitors were continued during the course of the disease in COVID-19 patients, as the use of RAS inhibitors was only established via medical record review or medical database review in majority of the studies included. Furthermore, there were some studies in which the duration of follow-up may not have been long enough for the outcomes of interest (mortality and/or severe/critical illness) to occur.

\section{Conclusions}

For patients with or without hypertension regardless of the treatment setting, it can be concluded from our metaanalyses that the use of RAS inhibitors would not increase the risk of mortality or severity in COVID-19. Indeed, the risk, if any, is outweighed by the benefits of continuing RAS inhibitors in COVID-19 patients with clinical indications where the efficacy of RAS inhibitors has been rigorously 
established. However, due to the variable quality of the existing evidence, this needs further confirmation from a methodologically sound, large, prospective cohort study or a randomized controlled trial.

Author contributions Conceptualization: SSH and CSK. Data curation: SSH and C.S.K. Formal analysis: SSH and CSK. Investigation: SSH, CSK, STRZ, HAM, and MAH. Methodology: SSH, CSK, STRZ, HAM, and MAH. Project administration: SSH and CSK. Resources: SSH and CSK. Visualization: SSH and CSK. Writing-original draft: SSH, HAM, and CSK. Writing-review and editing: SSH, CSK, STRZ, HAM, and MAH.

Availability of Data and Material Not applicable.

\section{Declarations}

Funding No external funding was used in the preparation of this article.

Conflict of interest Syed Shahzad Hasan, Chia Siang Kow, Muhammad Abdul Hadi, Syed Tabish Razi Zaidi, and Hamid A. Merchant declare that they have no potential conflicts of interest that might be relevant to the contents of this article.

Ethics approval Not applicable.

Consent to participate Not applicable.

Consent for publication Not applicable.

Code availability Not applicable.

\section{References}

1. Guan WJ, Ni ZY, Hu Y, et al. Clinical characteristics of coronavirus disease 2019 in China. N Engl J Med. 2020;382(18):1708-1720.

2. Zhang JJ, Dong X, Cao YY, et al. Clinical characteristics of 140 patients infected with SARS-CoV-2 in Wuhan, China. Allergy. 2020;75(7):1730-1741.

3. Zhou F, Yu T, Du R, et al. Clinical course and risk factors for mortality of adult inpatients with COVID-19 in Wuhan, China: a retrospective cohort study [published correction appears in Lancet. 2020 Mar 28;395(10229):1038] [published correction appears in Lancet. 2020 Mar 28;395(10229):1038]. Lancet. 2020;395(10229):1054-1062.

4. Wu C, Chen X, Cai Y, et al. Risk factors associated with acute respiratory distress syndrome and death in patients with coronavirus disease 2019 pneumonia in Wuhan, China. JAMA Intern Med. 2020;180(7):1-11.

5. Shi S, Qin M, Shen B, et al. Association of cardiac injury with mortality in hospitalized patients with COVID-19 in Wuhan, China. JAMA Cardiol. 2020;5(7):802-10.

6. Guo T, Fan Y, Chen M, et al. Cardiovascular implications of fatal outcomes of patients with coronavirus disease 2019 (COVID19). JAMA Cardiol. 2020;5(7):1-8.

7. Zheng YY, Ma YT, Zhang JY, Xie X. COVID-19 and the cardiovascular system. Nat Rev Cardiol. 2020;17(5):259-60.

8. Fang L, Karakiulakis G, Roth M. Are patients with hypertension and diabetes mellitus at increased risk for COVID-19 infection?
Lancet Respir Med. 2020;8(4):e21 [published correction appears in Lancet Respir Med. 2020 Jun; 8(6):e54].

9. Zhou P, Yang XL, Wang XG, et al. A pneumonia outbreak associated with a new coronavirus of probable bat origin. Nature. 2020;579(7798):270-73.

10. Ishiyama Y, Gallagher PE, Averill DB, Tallant EA, Brosnihan $\mathrm{KB}$, Ferrario CM. Upregulation of angiotensin-converting enzyme 2 after myocardial infarction by blockade of angiotensin II receptors. Hypertension. 2004;43(5):970-6.

11. Ocaranza MP, Godoy I, Jalil JE, et al. Enalapril attenuates downregulation of Angiotensin-converting enzyme 2 in the late phase of ventricular dysfunction in myocardial infarcted rat. Hypertension. 2006;48(4):572-8.

12. Soler MJ, Ye M, Wysocki J, William J, Lloveras J, Batlle D. Localization of ACE2 in the renal vasculature: amplification by angiotensin II type 1 receptor blockade using telmisartan. Am J Physiol Renal Physiol. 2009;296(2):F398-405.

13. Ferrario CM, Jessup J, Chappell MC, et al. Effect of angiotensinconverting enzyme inhibition and angiotensin II receptor blockers on cardiac angiotensin-converting enzyme 2. Circulation. 2005;111(20):2605-10.

14. Zhong JC, Ye JY, Jin HY, et al. Telmisartan attenuates aortic hypertrophy in hypertensive rats by the modulation of ACE2 and profilin-1 expression. Regul Pept. 2011;166(1-3):90-7.

15. Klimas J, Olvedy M, Ochodnicka-Mackovicova K, et al. Perinatally administered losartan augments renal ACE2 expression but not cardiac or renal Mas receptor in spontaneously hypertensive rats. J Cell Mol Med. 2015;19(8):1965-74.

16. Furuhashi M, Moniwa N, Mita T, et al. Urinary angiotensinconverting enzyme 2 in hypertensive patients may be increased by olmesartan, an angiotensin II receptor blocker. Am J Hypertens. 2015;28(1):15-21.

17. Vuille-dit-Bille RN, Camargo SM, Emmenegger L, et al. Human intestine luminal ACE2 and amino acid transporter expression increased by ACE-inhibitors. Amino Acids. 2015;47(4):693-705.

18. Burrell LM, Risvanis J, Kubota E, et al. Myocardial infarction increases ACE2 expression in rat and humans. Eur Heart J. 2005;26(4):324-69.

19. Burchill LJ, Velkoska E, Dean RG, Griggs K, Patel SK, Burrell LM. Combination renin-angiotensin system blockade and angiotensin-converting enzyme 2 in experimental myocardial infarction: implications for future therapeutic directions. Clin Sci (Lond). 2012;123(11):649-58.

20. Walters TE, Kalman JM, Patel SK, Mearns M, Velkoska E, Burrell LM. Angiotensin converting enzyme 2 activity and human atrial fibrillation: increased plasma angiotensin converting enzyme 2 activity is associated with atrial fibrillation and more advanced left atrial structural remodelling. Europace. 2017;19(8):1280-7.

21. Ramchand J, Patel SK, Srivastava PM, Farouque O, Burrell LM. Elevated plasma angiotensin converting enzyme 2 activity is an independent predictor of major adverse cardiac events in patients with obstructive coronary artery disease. PLoS One. 2018;13(6):e0198144.

22. Epelman S, Shrestha K, Troughton RW, et al. Soluble angiotensin-converting enzyme 2 in human heart failure: relation with myocardial function and clinical outcomes. J Card Fail. 2009;15(7):565-71.

23. Ramchand J, Patel SK, Kearney LG, et al. Plasma ACE2 activity predicts mortality in aortic stenosis and is associated with severe myocardial fibrosis. JACC Cardiovasc Imaging. 2020;13(3):655-64.

24. Kuba K, Imai Y, Rao S, et al. A crucial role of angiotensinconverting enzyme 2 (ACE2) in SARS coronavirus-induced lung injury. Nat Med. 2005;11(8):875-9. 
25. Imai Y, Kuba K, Rao S, et al. Angiotensin-converting enzyme 2 protects from severe acute lung failure. Nature. 2005;436(7047):112-6.

26. Zhang H, Baker A. Recombinant human ACE2: acing out angiotensin II in ARDS therapy. Crit Care. 2017;21(1):305.

27. Kow CS, Zaidi STR, Hasan SS. Cardiovascular disease and use of renin-angiotensin system inhibitors in COVID-19. Am J Cardiovasc Drugs. 2020;20(3):217-221.

28. Arentz M, Yim E, Klaff L, et al. Characteristics and outcomes of 21 critically Ill patients with COVID-19 in Washington State. JAMA. 2020;323(16):1612-4.

29. Bhatraju PK, Ghassemieh BJ, Nichols M, et al. Covid-19 in critically Ill patients in the Seattle Region-case series. N Engl J Med. 2020;382(21):2012-2022.

30. Grasselli G, Zangrillo A, Zanella A, et al. Baseline characteristics and outcomes of 1591 patients infected with SARSCoV-2 admitted to ICUs of the Lombardy Region. Italy. JAMA. 2020;323(16):1574-81.

31. Huang C, Wang Y, Li X, et al. Clinical features of patients infected with 2019 novel coronavirus in Wuhan, China. Lancet. 2020;395(10223):497-506.

32. Yang X, Yu Y, Xu J, et al. Clinical course and outcomes of critically ill patients with SARS-CoV-2 pneumonia in Wuhan, China: a single-centered, retrospective, observational study [published online ahead of print, $2020 \mathrm{Feb}$ 24]. Lancet Respir Med. 2020;8(4):e26.

33. Wang D, Hu B, Hu C, et al. Clinical characteristics of 138 hospitalized patients with 2019 novel coronavirus-infected pneumonia in Wuhan, China. JAMA. 2020;323(11):1061-9.

34. Chen N, Zhou M, Dong X, et al. Epidemiological and clinical characteristics of 99 cases of 2019 novel coronavirus pneumonia in Wuhan, China: a descriptive study. Lancet. 2020;395(10223):507-13.

35. Amaouche S, Letaief Z, Buls N, Allard S, Mey Jd. The relation between ACEI/ARB use and COVID-19 severity in RT-PCRconfirmed cases: A retrospective case-control study. Preprint. Research Square. 2020.

36. Chen C, Wang F, Chen P, et al. Mortality and pre-hospitalization use of Renin-Angiotensin System Inhibitors in hypertensive COVID-19 patients. J Am Heart Assoc. 2020. https://doi. org/10.1161/JAHA.120.017736

37. Chen M, Fan Y, Wu X, et al. Clinical characteristics and risk factors for fatal outcome in patients with 2019-Coronavirus Infected Disease (COVID-19) in Wuhan, China. Preprint. SSRN Electron J. 2020

38. Chen Y, Yang D, Cheng B, et al. Clinical characteristics and outcomes of patients with diabetes and COVID-19 in association with glucose-lowering medication. Diabetes Care. 2020;43(7):1399-407.

39. Feng Y, Ling Y, Bai T, et al. COVID-19 with different severities: a multicenter study of clinical features. Am J Respir Crit Care Med. 2020;201(11):1380-8.

40. Feng Z, Li J, Yao S, et al. The use of adjuvant therapy in preventing progression to severe pneumonia in patients with coronavirus disease 2019: a multicenter data analysis. Preprint. medRxiv. 2020; 2020.04.08.20057539.

41. Gao C, Cai Y, Zhang K, et al. Association of hypertension and antihypertensive treatment with COVID-19 mortality: a retrospective observational study. Eur Heart J. 2020;41(22):2058-66.

42. Guo T, Fan Y, Chen M, et al. Cardiovascular implications of fatal outcomes of patients with Coronavirus Disease 2019 (COVID19) [published online ahead of print, 2020 Mar 27]. JAMA Cardiol. 2020;5(7):1-8.

43. Hu J, Zhang X, Zhang X, et al. COVID-19 patients with hypertension have more severity condition, and ACEI/ARB treatment have no influence on the clinical severity and outcome [published online ahead of print, 2020 May 28]. J Infect. 2020;S0163-4453(20)30334-0.

44. Huang Z, Cao J, Yao Y, et al. The effect of RAS blockers on the clinical characteristics of COVID-19 patients with hypertension. Ann Transl Med. 2020;8(7):430.

45. Li J, Wang X, Chen J, Zhang H, Deng A. Association of reninangiotensin system inhibitors with severity or risk of death in patients with hypertension hospitalized for Coronavirus Disease 2019 (COVID-19) infection in Wuhan, China. JAMA Cardiol. 2020;5(7):1-6.

46. Li X, Xu S, Yu M, et al. Risk factors for severity and mortality in adult COVID-19 inpatients in Wuhan. J Allergy Clin Immunol. 2020;146(1):110-8.

47. Liu Y, Huang F, Xu J, et al. Anti-hypertensive Angiotensin II receptor blockers associated to mitigation of disease severity in elderly COVID-19 patients. Preprint. medRxiv. 2020;2020.03.20.20039586.

48. Meng J, Xiao G, Zhang J, et al. Renin-angiotensin system inhibitors improve the clinical outcomes of COVID-19 patients with hypertension. Emerg Microbes Infect. 2020;9(1):757-60.

49. Peng YD, Meng K, Guan HQ, et al. Clinical features and outcome of 112 cases of novel coronavirus pneumonia in cardiovascular patients [Chinese]. Chin. J. Cardiol. 2020;48.

50. Tan ND, Qiu Y, Xing XB, Ghosh S, Chen MH, Mao R. Associations between angiotensin-converting enzyme inhibitors and Angiotensin II Receptor blocker use, gastrointestinal symptoms, and mortality among patients with COVID-19. Gastroenterology. 2020;S0016-5085(20)30662-4.

51. Xu J, Huang C, Fan G, et al. Use of angiotensin-converting enzyme inhibitors and angiotensin II receptor blockers in context of COVID-19 outbreak: a retrospective analysis. Front Med. 2020;1-12.

52. Yan H, Valdes AM, Vijay A, et al. Role of drugs affecting the renin-angiotensin-aldosterone system on susceptibility and severity of COVID-19: a large case-control study from Zheijang Province, China. Preprint. medRxiv. 2020;2020.04.24.20077875.

53. Yang G, Tan Z, Zhou L, et al. Effects of angiotensin II receptor blockers and ACE (Angiotensin-Converting Enzyme) inhibitors on virus infection, inflammatory status, and clinical outcomes in patients with COVID-19 and hypertension: a single-center retrospective study. Hypertension. 2020;76(1):51-8.

54. Yuan Y, Liu D, Zeng S, et al. In-hospital Use of ACEI/ARB is associated with lower Risk of Mortality and Critic Illness in COVID-19 Patients with Hypertension: ACEI/ARB protect COVID-19 patients [published online ahead of print, 2020 Aug 12]. J Infect. 2020;S0163-4453(20)30548-X.

55. Zeng Z, Sha T, Zhang $Y$ et al. Hypertension in patients hospitalized with COVID-19 in Wuhan, China: a singlecenter retrospective observational study. Preprint. medRxiv. 2020;2020.04.06.20054825.

56. Zhang P, Zhu L, Cai J, et al. Association of inpatient use of angiotensin-converting enzyme inhibitors and angiotensin II receptor blockers with mortality among patients with hypertension hospitalized with COVID-19. Circ Res. 2020;126(12):1671-81.

57. Zhou F, Liu YM, Xie J, et al. Comparative impacts of ACE (Angiotensin-Converting Enzyme) inhibitors versus angiotensin ii receptor blockers on the risk of COVID-19 mortality. Hypertension. 2020;76(2):e15-7.

58. Zhou X, Zhu J, Xu T. Clinical characteristics of coronavirus disease 2019 (COVID-19) patients with hypertension on renin-angiotensin system inhibitors. Clin Exp Hypertens. 2020;42(7):656-60.

59. Fosbøl EL, Butt JH, Østergaard L, et al. Association of angiotensin-converting enzyme inhibitor or angiotensin receptor blocker use with COVID-19 diagnosis and mortality [published online ahead of print, 2020 Jun 19]. JAMA. 2020;324(2):168-77. 
60. Dauchet L, Lambert M, Gauthier V, et al, on behalf of the Lille COVID-19 study group. ACE inhibitors, AT1 receptor blockers and COVID-19: clinical epidemiology evidences for a continuation of treatments. The ACER-COVID study. Preprint. medRxiv 2020;2020.04.28.20078071.

61. Liabeuf S, Moragny J, Bennis Y, et al. Association between renin-angiotensin system inhibitors and COVID-19 complications [published online ahead of print, 2020 Jun 12]. Eur Heart J Cardiovasc Pharmacother. 2020; pvaa062.

62. Oussalah A, Gleye S, Clerc Urmes I, et al. Long-term ACE inhibitor/ARB use is associated with severe renal dysfunction and acute kidney injury in patients with severe COVID-19: results from a Referral Center Cohort in the North East of France [published online ahead of print, 2020 Jul 5]. Clin Infect Dis. 2020; ciaa677.

63. Zhou J, Tse G, Lee S, et al. Identifying main and interaction effects of risk factors to predict intensive care admission in patients hospitalized with COVID-19: a retrospective cohort study in Hong Kong. Preprint. medRxiv. 2020;2020.06.30.20143651.

64. Cannata F, Chiarito M, Reimers B, et al. Continuation versus discontinuation of ACE inhibitors or angiotensin II receptor blockers in COVID-19: effects on blood pressure control and mortality [published online ahead of print, 2020 Jun 5]. Eur Heart J Cardiovasc Pharmacother. 2020;pvaa056.

65. Conversano A, Melillo F, Napolano A, et al. Renin-angiotensin-aldosterone system inhibitors and outcome in patients with SARS-CoV-2 pneumonia: a case series study. Hypertension. 2020;76(2):e10-2.

66. Felice C, Nardin C, Di Tanna GL, et al. Use of RAAS inhibitors and risk of clinical deterioration in COVID-19: results from an Italian cohort of 133 hypertensives. Am J Hypertens. 2020;hpaa096. https://doi.org/10.1093/ajh/hpaa096

67. Ferrante G, Fazzari F, Cozzi O, et al. Risk factors for myocardial injury and death in patients with COVID-19: insights from a cohort study with chest computed tomography. Cardiovasc Res. 2020;cvaa193. https://doi.org/10.1093/cvr/cvaa193

68. Giacomelli A, Ridolfo AL, Milazzo L, et al. 30-day mortality in patients hospitalized with COVID-19 during the first wave of the Italian epidemic: a prospective cohort study. Pharmacol Res. 2020;158:104931.

69. Grasselli G, Greco M, Zanella A, et al. Risk Factors Associated With Mortality Among Patients With COVID-19 in Intensive Care Units in Lombardy, Italy [published online ahead of print, 2020 Jul 15]. JAMA Intern Med. 2020;e203539.

70. Rossi PG, Marino M, Formisano D, et al. Characteristics and outcomes of a cohort of SARS-CoV-2 patients in the Province of Reggio Emilia, Italy. Preprint. medRxiv. 2020;2020.04.13.20063545.

71. Sardu C, Maggi P, Messina V, et al. Could anti-hypertensive drug therapy affect the clinical prognosis of hypertensive patients with COVID-19 infection? Data from centers of southern Italy [published online ahead of print, 2020 Jul 7]. J Am Heart Assoc. 2020; 016948 .

72. Tedeschi S, Giannella M, Bartoletti M, et al. Clinical impact of renin-angiotensin system inhibitors on in-hospital mortality of patients with hypertension hospitalized for COVID-19 [published online ahead of print, 2020 Apr 27]. Clin Infect Dis. 2020; ciaa492.

73. Ayed M, Borahmah A, Yazdani A, Sultan A, Mossad A, Rawdhan H. Assessment of clinical characteristics and mortality-associated factors in COVID-19 Critical cases in Kuwait. Preprint. medRxiv. 2020;2020.06.17.20134007.

74. Dalan R, Ang LW, Tan WYT, et al. The association of hypertension and diabetes pharmacotherapy with COVID-19 severity and immune signatures: an observational study [published online ahead of print, 2020 Aug 7]. Eur Heart J Cardiovasc Pharmacother. 2020; pvaa098.

75. Choi HK, Koo H, Seok H, et al. ARB/ACEI use and severe COVID-19: a nationwide case-control study. Preprint. medRxiv. 2020;2020.06.12.20129916.

76. Jung SY, Choi JC, You SH, Kim WY. Association of renin-angiotensin-aldosterone system inhibitors with COVID-19-related outcomes in Korea: a nationwide population-based cohort study. Clin Infect Dis. 2020;ciaa624.

77. Lee H, Ahn J, Kang CK, et al. Association of angiotensin II receptor blockers and angiotensin-converting enzyme inhibitors on COVID-19-related outcome. Preprint. 2020. SSRN Electron J. 2020.

78. Son M, Seo J, Yang S. Association between Renin-AngiotensinAldosterone System Inhibitors and COVID-19 infection in South Korea. Hypertension. 2020;76(3):742-9.

79. Amat-Santos IJ, Santos-Martinez S, López-Otero D, et al. Ramipril in high-risk patients with COVID-19. J Am Coll Cardiol. 2020;76(3):268-76.

80. López-Otero D, López-Pais J, Cacho-Antonio CE, et al. Impact of angiotensin-converting enzyme inhibitors and angiotensin receptor blockers on COVID-19 in a western population. CARDIOVID registry. Rev Esp Cardiol (Engl Ed). 2020;S1885-5857(20)30224-3.

81. Selçuk M, Çınar T, Keskin M, et al. Is the use of ACE inb/ARBs associated with higher in-hospital mortality in Covid-19 pneumonia patients? Clin Exp Hypertens. 2020;42(8):738-42.

82. Baker KF, Hanrath AT, van der Loeff IS, et al. COVID-19 management in a UK NHS Foundation Trust with a High Consequence Infectious Diseases centre: a detailed descriptive analysis. Preprint. medRxiv. 2020;2020.05.14.20100834.

83. Argenziano MG, Bruce SL, Slater CL, et al. Characterization and clinical course of 1000 patients with coronavirus disease 2019 in New York: retrospective case series. BMJ. 2020;369:m1996.

84. Chang TS, Ding Y, Freund MK, et al. Prior diagnoses and medications as risk factors for COVID-19 in a Los Angeles Health System. Preprint. medRxiv. 2020;2020.07.03.20145581.

85. Chaudhri I, Koraishy FM, Bolotova O. Outcomes associated with the use of RAAS Blockade in hospitalized patients with SARSCoV-2 infection. Kidney360. 2020;1(8)801-809.

86. Imam Z, Odish F, Gill I, et al. Older age and comorbidity are independent mortality predictors in a large cohort of 1305 COVID-19 patients in Michigan, United States. J Intern Med. 2020. https://doi.org/10.1111/joim.13119.

87. Ip A, Parikh K, Parrillo JE, et al. Hypertension and Renin-Angiotensin-Aldosterone System Inhibitors in Patients with Covid-19. Preprint. medRxiv. 2020;2020.04.24.20077388.

88. Khera R, Clark C, Lu Y, et al. Association of angiotensinconverting enzyme inhibitors and angiotensin receptor blockers with the risk of hospitalization and death in hypertensive patients with coronavirus disease-19. Preprint. medRxiv. 2020; 2020.05.17.20104943.

89. Lam KW, Chow KW, Vo J, et al. Continued in-hospital ACE inhibitor and ARB Use in hypertensive COVID-19 patients is associated with positive clinical outcomes [published online ahead of print, 2020 Jul 23]. J Infect Dis. 2020; jiaa447.

90. Mehta N, Kalra A, Nowacki AS, et al. Association of use of Angiotensin-Converting Enzyme Inhibitors and Angiotensin II Receptor blockers with testing positive for coronavirus disease 2019 (COVID-19). JAMA Cardiol. 2020;e201855. https://doi. org/10.1001/jamacardio.2020.1855

91. Richardson S, Hirsch JS, Narasimhan M, et al. Presenting characteristics, comorbidities, and outcomes among 5700 patients hospitalized with COVID-19 in the New York City Area. JAMA. 2020;323(20):2052-9. 
92. Shah P, Owens J, Franklin J, Jani Y, Kumar A, Doshi R. Baseline use of angiotensin-converting enzyme inhibitor/AT1 blocker and outcomes in hospitalized coronavirus disease 2019 AfricanAmerican patients. J Hypertens. 2020. https://doi.org/10.1097/ HJH.0000000000002584.

93. Jung C, Bruno RR, Wernly B, et al. Inhibitors of the ReninAngiotensin-Aldosterone System and Covid-19 in critically ill elderly patients [published online ahead of print, $2020 \mathrm{Jul}$ 9]. Eur Heart J Cardiovasc Pharmacother. 2020; pvaa083.

94. Moher D, Liberati A, Tetzlaff J, Altman DG, PRISMA Group. Preferred reporting items for systematic reviews and meta-analyses: the PRISMA statement. BMJ. 2009;339:b2535.

95. Wells G, Shea B, O'Connell D, Peterson J, Welch V, Losos M, Tugwell P. The Newcastle-Ottawa Scale (NOS) for assessing the quality of nonrandomised studies in meta-analyses. 2013. http://www.ohri.ca/programs/clinical_epidemiology/oxford.asp. Accessed 28 Apr 282020.

96. Grover A, Oberoi M. A systematic review and meta-analysis to evaluate the clinical outcomes in COVID-19 patients on angiotensin-converting enzyme inhibitors or angiotensin receptor blockers [published online ahead of print, 2020 Jun 15]. Eur Heart J Cardiovasc Pharmacother. 2020; pvaa064.

97. Alamer A, Abraham I. Mortality in COVID-19 patients treated with ACEIs/ARBs: Re-estimated meta-analysis results following the Mehra et al. retraction [published online ahead of print, 2020 Jun 30]. Pharmacol Res. 2020;160:105053.

98. Greco A, Buccheri S, D'Arrigo P, et al. Outcomes of renin-angiotensin-aldosterone system blockers in patients with COVID-19: a systematic review and meta-analysis [published online ahead of print, 2020 Jul 16]. Eur Heart J Cardiovasc Pharmacother. 2020; pvaa074.

99. Pranata R, Permana H, Huang I, et al. The use of renin angiotensin system inhibitor on mortality in patients with coronavirus disease 2019 (COVID-19): A systematic review and meta-analysis [published online ahead of print, 2020 Jun 27]. Diabetes Metab Syndr. 2020;14(5):983-90.

100. Usman MS, Siddiqi TJ, Khan MS, et al. A Meta-analysis of the Relationship Between Renin-Angiotensin-Aldosterone System Inhibitors and COVID-19 [published online ahead of print, 2020 Jun 2]. Am J Cardiol. 2020;S0002-9149(20)30548-8.

101. Zhang X, Yu J, Pan LY, Jiang HY. ACEI/ARB use and risk of infection or severity or mortality of COVID-19: a systematic review and meta-analysis. Pharmacol Res. 2020;158:104927.

102. Liu X, Long C, Xiong Q, et al. Association of angiotensin converting enzyme inhibitors and angiotensin II receptor blockers with risk of COVID-19, inflammation level, severity, and death in patients with COVID-19: A rapid systematic review and metaanalysis. Clin Cardiol. 2020. https://doi.org/10.1002/clc.23421.
103. Guo X, Zhu Y, Hong Y. Decreased mortality of COVID-19 with renin-angiotensin-aldosterone system inhibitors therapy in patients with hypertension: a meta-analysis. Hypertension. 2020;76(2):e13-4.

104. Hasan SS, Capstick T, Ahmed R, et al. Mortality in COVID-19 patients with acute respiratory distress syndrome and corticosteroids use: a systematic review and meta-analysis. Expert Rev Respir Med. 2020. https://doi.org/10.1080/17476348.2020.18043 65.

105. Kim J, Choi SM, Lee J, et al. Effect of renin-angiotensin system blockage in patients with acute respiratory distress syndrome: a Retrospective Case Control Study. Korean J Crit Care Med. 2017;32(2):154-63.

106. Cao Y, Li L, Feng Z, et al. Comparative genetic analysis of the novel coronavirus (2019-nCoV/SARS-CoV-2) receptor ACE2 in different populations. Cell Discov. 2020;6:11.

107. Deshotels MR, Xia H, Sriramula S, Lazartigues E, Filipeanu CM. Angiotensin II mediates angiotensin converting enzyme type 2 internalization and degradation through an angiotensin II type I receptor-dependent mechanism. Hypertension. 2014;64(6):13681375 (published correction appears in Hypertension. 2014 Dec;64(6):e8. Sriramula, Srinivas [added]).

108. Pirola CJ, Sookoian S. Estimation of Renin-Angiotensin-Aldosterone-System (RAAS)-Inhibitor effect on COVID-19 outcome: a meta-analysis. J Infect. 2020;81(2):276-81.

109. Barochiner J, Martínez R. Use of inhibitors of the renin-angiotensin system in hypertensive patients and COVID-19 severity: A systematic review and meta-analysis. J Clin Pharm Ther. 2020. https://doi.org/10.1111/jcpt.13246.

110. Flacco ME, Acuti Martellucci C, Bravi F, et al. Treatment with ACE inhibitors or ARBs and risk of severe/lethal COVID-19: a meta-analysis [published online ahead of print, $2020 \mathrm{Jul} 1$ ]. Heart. 2020; heartjnl-2020-317336.

111. Li B, Yang J, Zhao F, et al. Prevalence and impact of cardiovascular metabolic diseases on COVID-19 in China. Clin Res Cardiol. 2020;109(5):531-8.

112. Wang B, Li R, Lu Z, Huang Y. Does comorbidity increase the risk of patients with COVID-19: evidence from meta-analysis. Aging (Albany NY). 2020;12(7):6049-6057.

113. Zhang XJ, Qin JJ, Cheng X, et al. In-hospital use of statins is associated with a reduced risk of mortality among individuals with COVID-19. Cell Metab. 2020;32(2):176-187.e4.

114. Kow CS, Hasan SS. Meta-analysis of effectiveness of statins in patients with severe COVID-19 [published online ahead of print, 2020 Aug 12]. Am J Cardiol. 2020. https://doi.org/10.1016/j. amjcard.2020.08.004. 